CRYSTALLOGRAPHIC COMMUNICATIONS

ISSN 2056-9890

Received 12 June 2015

Accepted 2 July 2015

Edited by M. Weil, Vienna University of Technology, Austria

Keywords: crystal structure; group 13 metals; five-coordination; dmpu; space-demanding solvent

CCDC reference: 1410078

Supporting information: this article has supporting information at journals.iucr.org/e

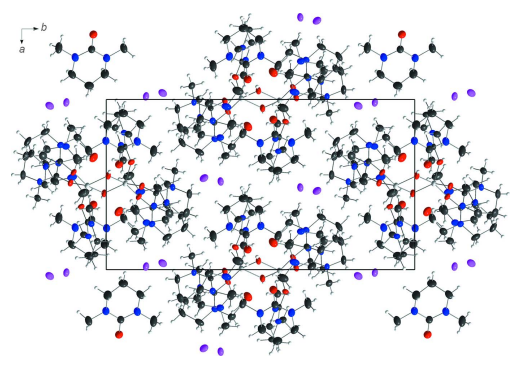

OPEN $\odot$ ACCESS

\section{Crystal structure of hexakis(dmpu)-di- $\mu_{2}$-hydroxido- dialuminium tetraiodide dmpu tetrasolvate [dmpu is 1,3-dimethyltetrahydropyrimidin-2(1H)-one]: a centrosymmetric dinuclear aluminium complex containing $\mathrm{AlO}_{5}$ polyhedra}

\author{
Daniel Lundberg ${ }^{\mathrm{a} *}$ and Krzysztof Lyczko ${ }^{\mathrm{b}}$
}

a Department of Chemistry and Biotechnology, PO Box 7015, Swedish University of Agricultural Sciences, S-750 07 Uppsala, Sweden, and ${ }^{\mathbf{b}}$ Institute of Nuclear Chemistry and Technology, Dorodna 16, PL-03-195 Warsaw, Poland. *Correspondence e-mail: daniel.lundberg@slu.se

The structure of the title compound, $\left[\mathrm{Al}_{2}(\mathrm{OH})_{2}\left(\mathrm{C}_{6} \mathrm{H}_{12} \mathrm{~N}_{2} \mathrm{O}\right)_{6}\right] \mathrm{I}_{4} \cdot 4 \mathrm{C}_{6} \mathrm{H}_{12} \mathrm{~N}_{2} \mathrm{O}$ (systematic name: di- $\mu_{2}$-hydroxido-bis $\{$ tris[1,3-dimethyltetrahydropyrimidin$2(1 H)$-one- $\kappa O$ ]aluminium $\}$ tetraiodide 1,3-dimethyltetrahydropyrimidin-2 $(1 H)$ one tetrasolvate), is composed of two $\mathrm{Al}\left(\mathrm{C}_{6} \mathrm{H}_{12} \mathrm{~N}_{2} \mathrm{O}\right)_{3}$ moieties linked into a centrosymmetric dinuclear unit by a pair of bridging hydroxide ions. The aluminium cations show a distorted trigonal bipyramidal $\mathrm{AlO}_{5}$ coordination environment formed only by monodentate ligands. The $\mathrm{Al}-\mathrm{O}$ bond lengths are in the range $1.789(2)-1.859$ (2) $\AA$ (mean bond length = $1.818 \AA$ ). The noncoordinating iodide anions compensate the charge of the complex cation. The remaining solvent molecules and the iodide counter-anions interact with the complex cation by weak non-classical $\mathrm{C}-\mathrm{H} \cdots \mathrm{I}$ and $\mathrm{C}-\mathrm{H} \cdots \mathrm{O}$ hydrogen bonds.

\section{Chemical context}

The solvent ligand $N, N^{\prime}$-dimethylpropyleneurea (dmpu; IUPAC name: 1,3-dimethyltetrahydropyrimidin-2(1H)-one, $\mathrm{C}_{6} \mathrm{H}_{12} \mathrm{~N}_{2} \mathrm{O}$ ) is known to be space-demanding upon coordination. This has been shown for several different metal ions which have a lower coordination number than the corresponding hydrates (Lundberg, 2006; Lundberg et al., 2010). In the boron group (group 13), the trivalent metal ions have previously been studied in dmpu solution and the solid state,

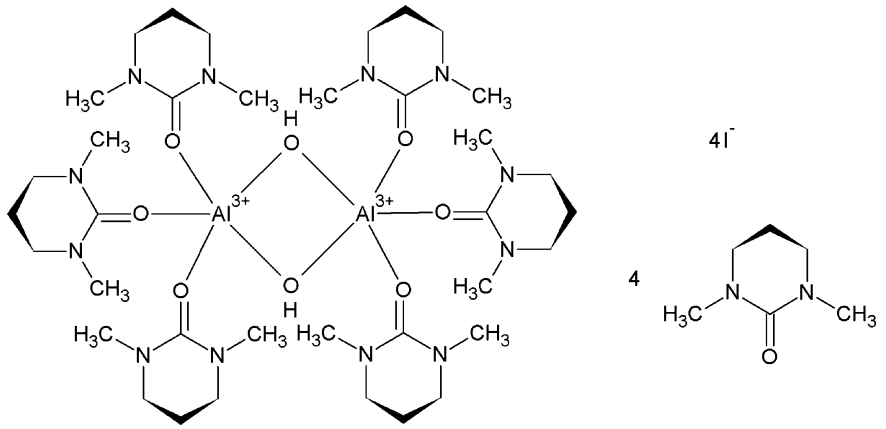

with reported crystal structures for trichloridobis(dmpu)thallium(III) (Carmalt et al., 1996) and tribromidobis(dmpu)indium(III) (Topel et al., 2010). In the case of dmpu-solvated gallium(III) bromide, the gallium cation was determined to be five-coordinate in solution but crystallization was not successful despite of repeated attempts (Topel et al., 2010). The title compound was prepared in an attempt to reveal the 
dmpu coordination for the last remaining naturally occurring trivalent group 13 metal ion, aluminium(III). Since both chloride and bromide ions are more prone to form aluminium complexes, the iodide salt was chosen as a starting material.

\section{Structural commentary}

The asymmetric unit of the title structure comprises one $\mathrm{Al}(\mathrm{dmpu})_{3}$ moiety, two dmpu solvent molecules and two iodide counter anions. The dinuclear cationic aluminium complex (Fig. 1) is generated by inversion symmetry and contains two five-coordinate aluminium cations, in which each cation is coordinated by the oxygen atoms of three dmpu ligand molecules and two $\mu_{2}$-bridging hydroxide ions, completing an $\mathrm{AlO}_{5}$ coordination sphere. The $\mathrm{Al}-\mathrm{O}$ bond lengths in the $\mathrm{Al}_{2}\left(\mu_{2}-\mathrm{OH}\right)_{2}$ bridge are 1.804 (2) and 1.859 (2) $\AA$, while the $\mathrm{Al}-\mathrm{O}$ bonds to the dmpu ligand molecules are 1.789 (2), 1.792 (2), and 1.846 (2) $\AA$, respectively. The two aluminium cations are separated by 2.883 (1) $\AA$ from each other. The $\mathrm{Al}-\mathrm{O}-\mathrm{C}$ angles for the coordinating dmpu ligand molecules lie in the range of 144.0 (2) to 154.7 (2) ${ }^{\circ}$. The dmpu ligand molecules are all essentially flat with the exception of the middle propylene carbon atom which is bent out of the plane with a dihedral angle of $c a 50^{\circ}$.

\section{Supramolecular features}

In the crystal packing, the complex cations are arranged in rods parallel to [001] with the counter-anions situated between the rods (Fig. 2). The hydroxide ion forms a medium-strength

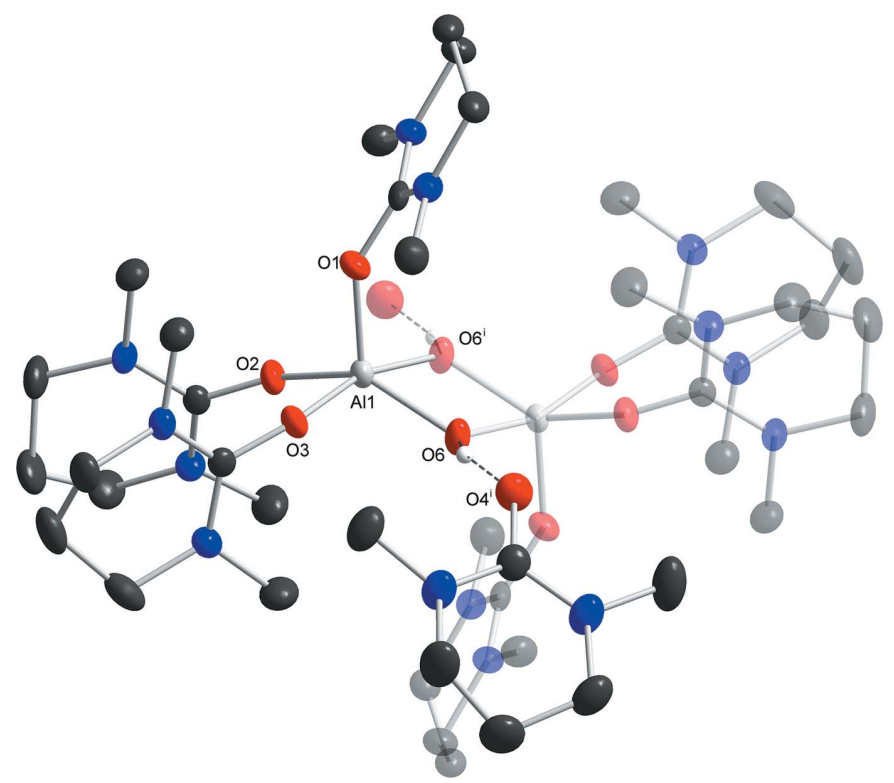

Figure 1

The dinuclear complex cation in the title compound, with displacement ellipsoids drawn at the $50 \%$ probability level. The hydrogen bonding from the bridging hydroxide group to the $\mathrm{O}$ atom $\left(\mathrm{O} 4^{\mathrm{i}}\right)$ of one noncoordinating dmpu molecule is indicated with a dashed line. Nonhydroxide $\mathrm{H}$ atoms have been omitted and the symmetry-related half of the complex has been shaded for clarity. [Symmetry code: (i) $-x, 1-y$, $1-z$.
Table 1

Hydrogen-bond geometry $\left(\AA{ }^{\circ}\right)$.

\begin{tabular}{|c|c|c|c|c|}
\hline$D-\mathrm{H} \cdots A$ & $D-\mathrm{H}$ & $\mathrm{H} \cdots A$ & $D \cdots A$ & $D-\mathrm{H} \cdots A$ \\
\hline $\mathrm{O} 6-\mathrm{H} 6 \cdots \mathrm{O} 4^{\mathrm{i}}$ & $0.73(5)$ & $1.91(5)$ & $2.625(3)$ & $167(5)$ \\
\hline $\mathrm{C} 5-\mathrm{H} 5 B \cdots \mathrm{I} 2$ & 0.98 & 3.01 & $3.987(3)$ & 172 \\
\hline $\mathrm{C} 6-\mathrm{H} 6 B \cdots \mathrm{O} 5^{\mathrm{ii}}$ & 0.98 & 2.21 & $3.190(4)$ & 174 \\
\hline $\mathrm{C} 12-\mathrm{H} 12 A \cdots \mathrm{O} 1$ & 0.98 & 2.59 & $3.561(4)$ & 173 \\
\hline $\mathrm{C} 12-\mathrm{H} 12 B \cdots \mathrm{I} 1^{\mathrm{iii}}$ & 0.98 & 3.09 & $4.051(3)$ & 167 \\
\hline $\mathrm{C} 14-\mathrm{H} 14 A \cdots \mathrm{I} 2^{\mathrm{iv}}$ & 0.99 & 3.15 & $4.070(4)$ & 156 \\
\hline $\mathrm{C} 17-\mathrm{H} 17 B \cdots \mathrm{I} 1^{\mathrm{iv}}$ & 0.98 & 3.05 & 4.015 (4) & 169 \\
\hline $\mathrm{C} 16-\mathrm{H} 16 A \cdots \mathrm{I} 1^{\mathrm{iii}}$ & 0.99 & 3.11 & $3.932(4)$ & 141 \\
\hline $\mathrm{C} 24-\mathrm{H} 24 A \cdots \mathrm{O} 3^{\mathrm{i}}$ & 0.98 & 2.57 & $3.482(5)$ & 154 \\
\hline $\mathrm{C} 28-\mathrm{H} 28 B \cdots \mathrm{I} 2^{\mathrm{v}}$ & 0.99 & 3.09 & 3.981 (4) & 150 \\
\hline $\mathrm{C} 30-\mathrm{H} 30 A \cdots \mathrm{O} 5^{\mathrm{vi}}$ & 0.98 & 2.57 & $3.404(5)$ & 143 \\
\hline
\end{tabular}

Symmetry codes: (i) $-x,-y+1,-z+1$; $\quad$ (ii) $-x+1,-y+1,-z+2$; $\quad$ (iii) $x-\frac{1}{2},-y+\frac{3}{2}, z+\frac{1}{2} ; \quad$ (iv) $\quad x-1, y, z ; \quad$ (v) $\quad x+\frac{1}{2},-y+\frac{3}{2}, z+\frac{1}{2}$; $\quad$ (vi) $-x+2,-y+1,-z+2$.

$\mathrm{O}-\mathrm{H}$. . O hydrogen bond of 2.625 (3) $\AA$ to one of the noncoordinating dmpu ligand molecules, with an $\mathrm{H}$. . O $-\mathrm{C}$ angle for this interaction of $134.8(17)^{\circ}$. The other non-coordinating dmpu molecule is stabilized by a much weaker $\mathrm{O} \cdots \mathrm{H}-\mathrm{C}$ interaction of $3.190(5) \AA$. Other $\mathrm{O} \cdots \mathrm{H}-\mathrm{C}$ interaction between the moieties range from 3.404 (5)-3.561 (4) A. The remaining positive charges on the aluminium atoms in the complex are compensated by the presence of non-coordin-

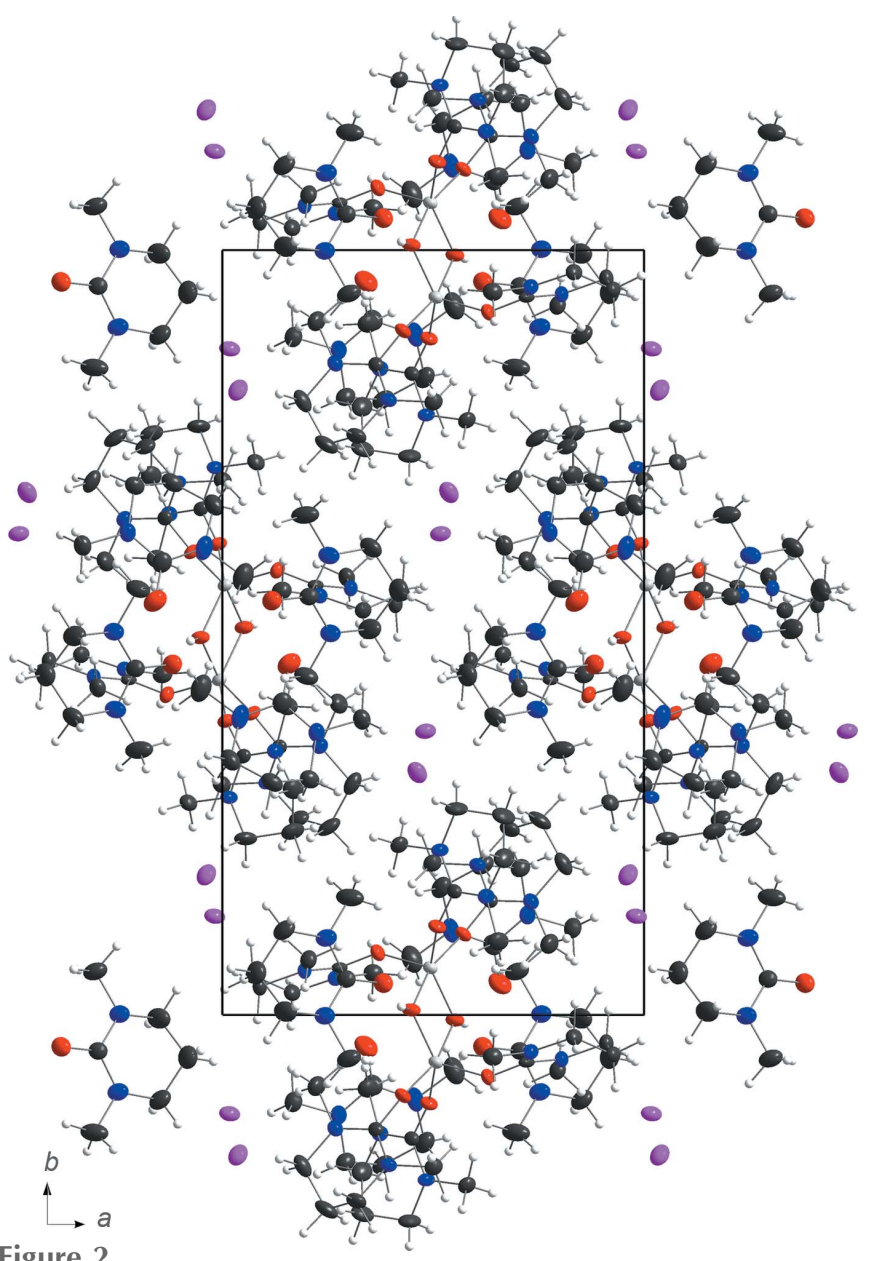

Figure 2

The crystal packing of the title structure in a view along [001]. 


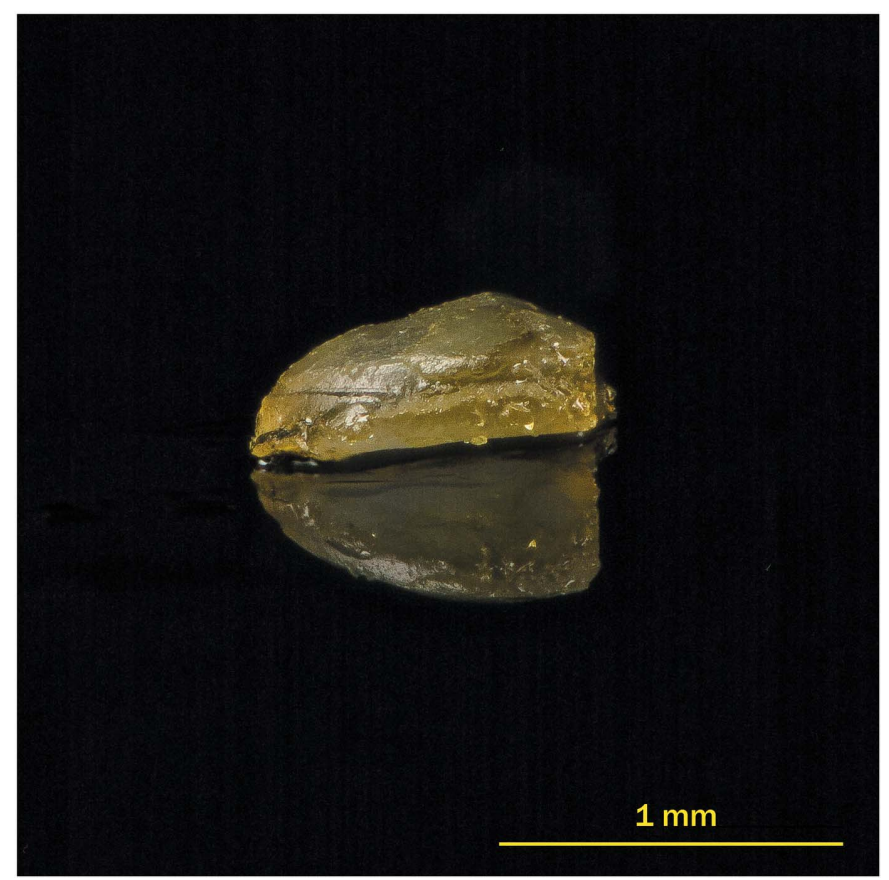

Figure 3

High-resolution photograph of another, partially crystalline sample of the title compound. Multiple exposures were stacked for an increased depth of field.

ating iodide anions, which interact with the cationic complex by weak $\mathrm{I} \cdots \mathrm{H}-\mathrm{C}$ hydrogen bonds in the range 3.932 (4)4.070 (4) А (Table 1).

\section{Database survey}

The Cambridge Structural Database (Version 2015; Groom \& Allen, 2014) lists 615 structures with an $\mathrm{AlO}_{4}$ coordination polyhedron and 387 structures with an $\mathrm{AlO}_{6}$ polyhedron, but only 46 with an $\mathrm{AlO}_{5}$ polyhedron. Of these 46, three contain $\mu_{2}$-hydroxido bridges, including two polynuclear structures (Abrahams et al., 2002; Murugavel \& Kuppuswamy, 2006) and a trinuclear structure with an $\mathrm{AlO}_{3} \mathrm{~N}_{2}-\mathrm{AlO}_{5}-\mathrm{AlO}_{3} \mathrm{~N}_{2}$ motif. Another trinuclear complex with an $\mathrm{AlO}_{4}-\mathrm{AlO}_{5}-\mathrm{AlO}_{4}$ motif, albeit without hydroxide bridges (Pauls \& Neumüller, 2000), and two different mononuclear, five-coordinate tetrahydrofuran (thf) solvates have been reported (Karsch et al., 2012). More than 50 examples of dimeric complexes with hexacoordinate aluminium ions with similar bridging between aluminium have been reported.

Urea solvated aluminium perchlorate was structurally determined by Mooy et al. (1974) as a hexacoordinate, homoleptic complex. Homoleptic hexacoordination is also found in other common, non-aqueous $O$-donor solvents, including dimethylsulfoxide (dmso) solvated aluminium chloride (Boström et al., 2003), hexaisothiocyanatoaluminium (Gumbris et al., 2012), iodide (Molla-Abbassi et al., 2003), and perchlorate (Chan et al., 2004), as well as $N, N$-dimethylformamide (dmf) solvated aluminium hexachloridotechnate chloride (Benz et al., 2015), perchlorate (Suzuki \& Ishiguro, 1998), and tribromide (Bekaert et al., 2002), and the $N, N$ -
Table 2

Experimental details.

\begin{tabular}{|c|c|}
\hline \multicolumn{2}{|l|}{ Crystal data } \\
\hline Chemical formula & $\begin{array}{l}{\left[\mathrm{Al}_{2}(\mathrm{OH})_{2}\left(\mathrm{C}_{6} \mathrm{H}_{12} \mathrm{~N}_{2} \mathrm{O}\right)_{6}\right] \mathrm{I}_{4}} \\
\quad{ }_{4 \mathrm{C}_{6} \mathrm{H}_{12} \mathrm{~N}_{2} \mathrm{O}}\end{array}$ \\
\hline$M_{\mathrm{r}}$ & 1877.33 \\
\hline Crystal system, space group & Monoclinic, $P 2_{1} / n$ \\
\hline Temperature $(\mathrm{K})$ & 100 \\
\hline$a, b, c(\AA)$ & $\begin{array}{l}13.9120(2), 22.6152(2), \\
14.4875(3)\end{array}$ \\
\hline$\beta\left({ }^{\circ}\right)$ & $116.331(2)$ \\
\hline$V\left(\AA^{3}\right)$ & $4085.16(12)$ \\
\hline$Z$ & 2 \\
\hline Radiation type & $\mathrm{Cu} K \alpha$ \\
\hline$\mu\left(\mathrm{mm}^{-1}\right)$ & 12.72 \\
\hline Crystal size (mm) & $0.20 \times 0.16 \times 0.14$ \\
\hline \multicolumn{2}{|l|}{ Data collection } \\
\hline Diffractometer & $\begin{array}{l}\text { Agilent SuperNova Dual Source } \\
\text { diffractometer with an Eos } \\
\text { detector }\end{array}$ \\
\hline Absorption correction & $\begin{array}{l}\text { Multi-scan (CrysAlis PRO; } \\
\text { Agilent, 2014) }\end{array}$ \\
\hline$T_{\min }, T_{\max }$ & $0.411,1.000$ \\
\hline $\begin{array}{l}\text { No. of measured, independent and } \\
\text { observed }[I>2 \sigma(I)] \text { reflections }\end{array}$ & $75993,7114,6779$ \\
\hline$R_{\text {int }}$ & 0.040 \\
\hline$(\sin \theta / \lambda)_{\max }\left(\AA^{-1}\right)$ & 0.593 \\
\hline \multicolumn{2}{|l|}{ Refinement } \\
\hline$R\left[F^{2}>2 \sigma\left(F^{2}\right)\right], w R\left(F^{2}\right), S$ & $0.030,0.078,1.10$ \\
\hline No. of reflections & 7114 \\
\hline No. of parameters & 456 \\
\hline $\mathrm{H}$-atom treatment & $\begin{array}{l}\mathrm{H} \text { atoms treated by a mixture of } \\
\text { independent and constrained } \\
\text { refinement }\end{array}$ \\
\hline$\Delta \rho_{\max }, \Delta \rho_{\min }\left(\mathrm{e} \AA^{-3}\right)$ & $1.20,-1.13$ \\
\hline
\end{tabular}

Computer programs: CrysAlis PRO (Agilent, 2014), SHELXS97 (Sheldrick, 2008), SHELXL2014 (Sheldrick, 2015) and DIAMOND (Crystal Impact, 2001).

dimethylacetamide (dma) solvated aluminium perchlorate (Suzuki \& Ishiguro, 2006). One homoleptic, tetracoordinate aluminium ion has been reported by Engesser et al. (2012) with an anionic $O$-donor ligand.

\section{Synthesis and crystallization}

The title compound was prepared by dissolving anhydrous aluminium(III) iodide (Sigma-Aldrich) in distilled dmpu in a glass vial, and subsequently heated in an oil bath to approximately $323 \mathrm{~K}$, and then allowed to cool while still in the oil bath. After cooling to room temperature, the sample was refrigerated $(277 \mathrm{~K})$ for several weeks to allow for crystal growth. The presence of hydroxide ions in the title compound was most likely caused during preparation of the mother liquor. It appears possible that with additional precautions, a hydroxide-free compound might be obtained. A part of the solid was photographed in detail at ambient room temperature (Fig. 3), whereas attempts to study smaller crystals failed, presumably due to the hygroscopicity of the material.

\section{Refinement}

Hydrogen atoms bonded to carbon atoms were placed in calculated positions with $\mathrm{C}-\mathrm{H}=0.98$ (methyl) or $0.99 \AA$ 
(methylene) and refined isotropically using a riding model with $U_{\text {iso }}(\mathrm{H})$ equal to $1.5 U_{\text {eq }}(\mathrm{C})$ or $1.2 U_{\text {eq }}(\mathrm{C})$ for methyl and methylene hydrogen atoms, respectively. The hydrogen atom of the hydroxide group was located in a difference map and its position and $U_{\text {iso }}$ value were freely refined. Crystal data, data collection and structure refinement details are summarized in Table 2.

\section{Acknowledgements}

We thank Ingmar Persson and Lars Eriksson for their interest in this work, and Harald Cederlund for obtaining the highresolution crystallophotography (HRCP) image.

\section{References}

Abrahams, I., Bradley, D. C., Chudzynska, H., Motevalli, M. \& Sinclair, R. A. (2002). J. Chem. Soc. Dalton Trans. pp. 259-266.

Agilent (2014). CrysAlis PRO. Agilent Technologies, Yarnton, England.

Bekaert, A., Barberan, O., Kaloun, E. B., Rabhi, C., Danan, A., Brion, J. D., Lemoine, P. \& Viossat, B. (2002). Z. Kristallogr. New Cryst. Struct. 217, 128-130.

Benz, M., Braband, H., Schmutz, P., Halter, J. \& Alberto, R. (2015). Chem. Sci. 6, 165-169.

Boström, D., Clausén, M. \& Sandström, M. (2003). Acta Cryst. E59, m934-m935.

Carmalt, C. J., Farrugia, L. J. \& Norman, N. C. (1996). Main Group Chem. 1, 339-344.
Chan, E. J., Cox, B. G., Harrowfield, J. M., Ogden, M. F., Skelton, B. W. \& White, A. H. (2004). Inorg. Chim. Acta, 357, 2365-2373.

Crystal Impact (2001). DIAMOND. Crystal Impact GbR, Bonn, Germany.

Engesser, T. A., Hrobarik, P., Trapp, N., Eiden, P., Scherer, H., Kaupp, M. \& Krossing, I. (2012). ChemPlusChem, 77, 643-651.

Groom, C. R. \& Allen, F. H. (2014). Angew. Chem. Int. Ed. 53, 662 671.

Gumbris, E. G., Peresypkina, E. V., Virovets, A. V. \& Cherkasova, T. G. (2012). Russ. J. Inorg. Chem. 57, 337-342.

Karsch, M., Lund, H., Schulz, A., Villinger, A. \& Voss, K. (2012). Eur. J. Inorg. Chem. pp. 5542-5553.

Lundberg, D. (2006). PhD thesis, Swedish University of Agricultural Sciences, Sweden. Available for free at http://pub.epsilon.slu.se/ 1072/.

Lundberg, D., Persson, I., Eriksson, L., D'Angelo, P. \& De Panfilis, S. (2010). Inorg. Chem. 49, 4420-4432.

Molla-Abbassi, A., Skripkin, M., Kritikos, M., Persson, I., Mink, J. \& Sandström, M. (2003). Dalton Trans. pp. 1746-1753.

Mooy, J. H. M., Krieger, W., Heijdenrijk, D. \& Stam, C. H. (1974). Chem. Phys. Lett. 29, 179-182.

Murugavel, R. \& Kuppuswamy, S. (2006). Angew. Chem. Int. Ed. 45, $7022-7026$.

Pauls, J. \& Neumüller, B. (2000). Z. Anorg. Allg. Chem. 626, 270-279.

Sheldrick, G. M. (2008). Acta Cryst. A64, 112-122.

Sheldrick, G. M. (2015). Acta Cryst. C71, 3-8.

Suzuki, H. \& Ishiguro, S.-I. (1998). Acta Cryst. C54, 586-588.

Suzuki, H. \& Ishiguro, S. (2006). Acta Cryst. E62, m576-m578.

Topel, Ö., Persson, I., Lundberg, D. \& Ullström, A.-S. (2010). Inorg. Chim. Acta, 363, 988-994. 


\section{supporting information}

Acta Cryst. (2015). E71, 895-898 [https://doi.org/10.1107/S2056989015012785]

Crystal structure of hexakis(dmpu)-di- $\mu_{2}$-hydroxido-dialuminium tetraiodide dmpu tetrasolvate [dmpu is 1,3-dimethyltetrahydropyrimidin-2(1H)-one]: a centrosymmetric dinuclear aluminium complex containing $\mathrm{AlO}_{5}$ polyhedra

\section{Daniel Lundberg and Krzysztof Lyczko}

Computing details

Data collection: CrysAlis PRO (Agilent, 2014); cell refinement: CrysAlis PRO (Agilent, 2014); data reduction: CrysAlis PRO (Agilent, 2014); program(s) used to solve structure: SHELXS97 (Sheldrick, 2008); program(s) used to refine structure: SHELXL2014 (Sheldrick, 2015); molecular graphics: DIAMOND (Crystal Impact, 2001); software used to prepare material for publication: SHELXL2014 (Sheldrick, 2015).

Di- $\mu_{2}$-hydroxido-bis $\{$ tris $[1,3$-dimethyltetrahydropyrimidin-2 $(1 \mathrm{H})$-one- $\kappa \mathrm{O}]$ aluminium\} tetraiodide 1,3dimethyltetrahydropyrimidin-2(1H)-one tetrasolvate

Crystal data

$\left[\mathrm{Al}_{2}(\mathrm{OH})_{2}\left(\mathrm{C}_{6} \mathrm{H}_{12} \mathrm{~N}_{2} \mathrm{O}\right)_{6}\right] \mathrm{I}_{4} \cdot 4 \mathrm{C}_{6} \mathrm{H}_{12} \mathrm{~N} 2 \mathrm{O}$

$M_{r}=1877.33$

Monoclinic, $P 2{ }_{1} / n$

$a=13.9120(2) \AA$

$b=22.6152(2) \AA$

$c=14.4875(3) \AA$

$\beta=116.331(2)^{\circ}$

$V=4085.16(12) \AA^{3}$

$Z=2$

\section{Data collection}

Agilent SuperNova Dual Source diffractometer with an Eos detector

Radiation source: SuperNova $(\mathrm{Cu}) \mathrm{X}$-ray Source

Detector resolution: 16.0131 pixels $\mathrm{mm}^{-1}$ $\omega$ scans

Absorption correction: multi-scan

(CrysAlis PRO; Agilent, 2014)

$T_{\min }=0.411, T_{\max }=1.000$

Refinement

Refinement on $F^{2}$

Least-squares matrix: full

$R\left[F^{2}>2 \sigma\left(F^{2}\right)\right]=0.030$

$w R\left(F^{2}\right)=0.078$

$S=1.10$
$F(000)=1912$

$D_{\mathrm{x}}=1.526 \mathrm{Mg} \mathrm{m}^{-3}$

$\mathrm{Cu} K \alpha$ radiation, $\lambda=1.54184 \AA$

Cell parameters from 30242 reflections

$\theta=3.9-69.2^{\circ}$

$\mu=12.72 \mathrm{~mm}^{-1}$

$T=100 \mathrm{~K}$

Block, yellow

$0.20 \times 0.16 \times 0.14 \mathrm{~mm}$

75993 measured reflections

7114 independent reflections

6779 reflections with $I>2 \sigma(I)$

$R_{\text {int }}=0.040$

$\theta_{\max }=66.0^{\circ}, \theta_{\min }=3.7^{\circ}$

$h=-16 \rightarrow 16$

$k=-26 \rightarrow 26$

$l=-17 \rightarrow 17$

7114 reflections

456 parameters

0 restraints

Hydrogen site location: mixed 
$\mathrm{H}$ atoms treated by a mixture of independent and constrained refinement

$w=1 /\left[\sigma^{2}\left(F_{\mathrm{o}}^{2}\right)+(0.0364 P)^{2}+6.5832 P\right]$

where $P=\left(F_{\mathrm{o}}^{2}+2 F_{\mathrm{c}}^{2}\right) / 3$

$$
\begin{aligned}
& (\Delta / \sigma)_{\max }=0.002 \\
& \Delta \rho_{\max }=1.20 \mathrm{e} \AA^{-3} \\
& \Delta \rho_{\min }=-1.13 \mathrm{e} \AA^{-3}
\end{aligned}
$$

Special details

Geometry. All e.s.d.'s (except the e.s.d. in the dihedral angle between two 1.s. planes) are estimated using the full covariance matrix. The cell e.s.d.'s are taken into account individually in the estimation of e.s.d.'s in distances, angles and torsion angles; correlations between e.s.d.'s in cell parameters are only used when they are defined by crystal symmetry.

\begin{tabular}{|c|c|c|c|c|}
\hline & $x$ & $y$ & $z$ & $U_{\text {iso }} * / U_{\text {eq }}$ \\
\hline I1 & $0.51772(2)$ & $0.62910(2)$ & $0.13190(2)$ & $0.03025(7)$ \\
\hline $\mathrm{I} 2$ & $0.53752(2)$ & $0.68312(2)$ & $0.63215(2)$ & $0.03565(8)$ \\
\hline Al1 & $0.00668(7)$ & $0.56180(4)$ & $0.52651(7)$ & $0.01970(18)$ \\
\hline $\mathrm{O} 1$ & $0.13114(16)$ & $0.58133(9)$ & $0.63291(16)$ & $0.0250(5)$ \\
\hline $\mathrm{O} 3$ & $-0.07158(16)$ & $0.60555(9)$ & $0.57705(16)$ & $0.0240(4)$ \\
\hline N9 & $0.7761(2)$ & $0.63074(12)$ & $0.9445(2)$ & $0.0298(6)$ \\
\hline N2 & $0.2309(2)$ & $0.54816(11)$ & $0.7944(2)$ & $0.0251(5)$ \\
\hline N1 & $0.3048(2)$ & $0.55782(11)$ & $0.6791(2)$ & $0.0252(5)$ \\
\hline $\mathrm{C} 1$ & $0.2217(2)$ & $0.56185(12)$ & $0.7019(2)$ & $0.0218(6)$ \\
\hline $\mathrm{O} 2$ & $-0.00991(17)$ & $0.61562(9)$ & $0.42976(17)$ & $0.0261(5)$ \\
\hline N4 & $-0.0161(2)$ & $0.71546(10)$ & 0.41492 (19) & $0.0230(5)$ \\
\hline N8 & $0.2568(2)$ & $0.49894(13)$ & $0.3074(2)$ & $0.0338(7)$ \\
\hline $\mathrm{C} 7$ & $-0.0504(2)$ & $0.66163(13)$ & $0.3756(2)$ & $0.0194(6)$ \\
\hline $\mathrm{O} 4$ & $0.11822(19)$ & $0.54165(10)$ & $0.32421(19)$ & $0.0339(5)$ \\
\hline $\mathrm{C} 12$ & 0.0803 & $0.72256(14)$ & $0.5114(2)$ & $0.0277(7)$ \\
\hline $\mathrm{H} 12 \mathrm{~A}$ & 0.0955 & 0.6856 & 0.5506 & $0.042 *$ \\
\hline H12B & 0.0694 & 0.7544 & 0.5516 & $0.042 *$ \\
\hline $\mathrm{H} 12 \mathrm{C}$ & 0.1408 & 0.7324 & 0.4969 & $0.042 *$ \\
\hline N5 & $-0.2321(2)$ & $0.64829(11)$ & $0.4770(2)$ & $0.0259(6)$ \\
\hline N6 & $-0.1056(2)$ & $0.69603(11)$ & $0.6212(2)$ & $0.0241(5)$ \\
\hline N3 & $-0.1258(2)$ & $0.65501(11)$ & 0.2794 & $0.0245(5)$ \\
\hline $\mathrm{C} 17$ & $-0.2762(3)$ & $0.59303(17)$ & 0.4251 & $0.0413(9)$ \\
\hline H17A & -0.2177 & 0.5654 & 0.4364 & $0.062 *$ \\
\hline H17B & -0.3179 & 0.6002 & 0.3511 & $0.062 *$ \\
\hline $\mathrm{H} 17 \mathrm{C}$ & -0.3230 & 0.5760 & 0.4526 & $0.062 *$ \\
\hline O5 & $0.8410(2)$ & $0.54111(12)$ & $1.0114(2)$ & $0.0441(6)$ \\
\hline $\mathrm{C} 13$ & $-0.1357(2)$ & $0.64952(13)$ & $0.5582(2)$ & $0.0201(6)$ \\
\hline $\mathrm{C} 5$ & $0.2954(3)$ & $0.57220(15)$ & $0.5773(3)$ & $0.0313(7)$ \\
\hline $\mathrm{H} 5 \mathrm{~A}$ & 0.3051 & 0.5362 & 0.5447 & $0.047^{*}$ \\
\hline H5B & 0.3505 & 0.6012 & 0.5840 & $0.047 *$ \\
\hline $\mathrm{H} 5 \mathrm{C}$ & 0.2242 & 0.5889 & 0.5349 & $0.047^{*}$ \\
\hline C6 & 0.1414 & $0.55467(15)$ & $0.8202(3)$ & $0.0311(7)$ \\
\hline H6A & 0.1458 & 0.5933 & 0.8525 & $0.047^{*}$ \\
\hline H6B & 0.1446 & 0.5233 & 0.8681 & $0.047^{*}$ \\
\hline $\mathrm{H} 6 \mathrm{C}$ & 0.0737 & 0.5518 & 0.7573 & $0.047 *$ \\
\hline
\end{tabular}
An approximate (isotropic) treatment of cell e.s.d.'s is used for estimating e.s.d.'s involving l.s. planes.

Fractional atomic coordinates and isotropic or equivalent isotropic displacement parameters $\left(\AA^{2}\right)$ 


\begin{tabular}{|c|c|c|c|c|}
\hline N7 & $0.2533(2)$ & 0.60067 (13) & $0.3315(2)$ & $0.0342(7)$ \\
\hline N10 & $0.9575(2)$ & $0.61058(15)$ & $1.0066(2)$ & $0.0411(7)$ \\
\hline $\mathrm{C} 20$ & $0.3530(3)$ & $0.61090(17)$ & $0.3229(3)$ & $0.0410(9)$ \\
\hline $\mathrm{H} 20 \mathrm{~A}$ & 0.3949 & 0.6425 & 0.3715 & $0.049^{*}$ \\
\hline H20B & 0.3360 & 0.6242 & 0.2523 & $0.049^{*}$ \\
\hline C18 & $0.0012(3)$ & 0.69860 (14) & $0.7087(3)$ & $0.0293(7)$ \\
\hline H18A & -0.0035 & 0.6866 & 0.7715 & $0.044^{*}$ \\
\hline H18B & 0.0288 & 0.7391 & 0.7167 & $0.044^{*}$ \\
\hline $\mathrm{H} 18 \mathrm{C}$ & 0.0497 & 0.6719 & 0.6963 & $0.044 *$ \\
\hline $\mathrm{C} 14$ & $-0.3091(3)$ & 0.69760 (17) & $0.4505(3)$ & $0.0367(8)$ \\
\hline H14A & -0.3642 & 0.6882 & 0.4741 & $0.044^{*}$ \\
\hline H14B & -0.3457 & 0.7027 & 0.3748 & $0.044^{*}$ \\
\hline $\mathrm{C} 8$ & $-0.1683(3)$ & 0.70498 (16) & $0.2085(3)$ & $0.0360(8)$ \\
\hline $\mathrm{H} 8 \mathrm{~A}$ & -0.2426 & 0.6963 & 0.1569 & $0.043^{*}$ \\
\hline H8B & -0.1241 & 0.7113 & 0.1714 & $0.043^{*}$ \\
\hline $\mathrm{C} 15$ & $-0.2530(3)$ & 0.75435 (16) & $0.5002(3)$ & $0.0403(9)$ \\
\hline H15A & -0.2094 & 0.7686 & 0.4661 & $0.048^{*}$ \\
\hline H15B & -0.3066 & 0.7851 & 0.4927 & $0.048^{*}$ \\
\hline $\mathrm{C} 27$ & $0.8817(3)$ & $0.69576(16)$ & $0.8932(3)$ & $0.0377(8)$ \\
\hline H27A & 0.8970 & 0.7377 & 0.8856 & $0.045^{*}$ \\
\hline H27B & 0.8598 & 0.6759 & 0.8260 & $0.045^{*}$ \\
\hline $\mathrm{C} 26$ & $0.7926(3)$ & $0.69190(15)$ & $0.9246(3)$ & $0.0328(7)$ \\
\hline $\mathrm{H} 26 \mathrm{~A}$ & 0.8109 & 0.7158 & 0.9874 & $0.039^{*}$ \\
\hline H26B & 0.7257 & 0.7080 & 0.8691 & $0.039^{*}$ \\
\hline $\mathrm{C} 3$ & $0.4264(3)$ & 0.55167 (14) & $0.8624(3)$ & $0.0293(7)$ \\
\hline $\mathrm{H} 3 \mathrm{~A}$ & 0.4940 & 0.5336 & 0.9132 & $0.035^{*}$ \\
\hline H3B & 0.4324 & 0.5951 & 0.8725 & $0.035^{*}$ \\
\hline C19 & $0.2059(3)$ & $0.54700(14)$ & $0.3204(3)$ & $0.0275(7)$ \\
\hline $\mathrm{C} 16$ & $-0.1821(3)$ & $0.74310(14)$ & $0.6123(3)$ & $0.0327(7)$ \\
\hline H16A & -0.1429 & 0.7797 & 0.6455 & $0.039^{*}$ \\
\hline H16B & -0.2260 & 0.7309 & 0.6472 & $0.039^{*}$ \\
\hline $\mathrm{C} 28$ & 0.9807 (3) & 0.66668 (17) & $0.9733(3)$ & $0.0413(9)$ \\
\hline $\mathrm{H} 28 \mathrm{~A}$ & 1.0326 & 0.6605 & 0.9446 & $0.050^{*}$ \\
\hline H28B & 1.0144 & 0.6933 & 1.0335 & $0.050^{*}$ \\
\hline $\mathrm{C} 25$ & $0.8574(3)$ & $0.59161(16)$ & 0.9887 (3) & $0.0329(7)$ \\
\hline $\mathrm{C} 29$ & $0.6688(3)$ & $0.61365(16)$ & $0.9271(3)$ & $0.0324(7)$ \\
\hline H29A & 0.6521 & 0.6318 & 0.9797 & $0.049^{*}$ \\
\hline H29B & 0.6649 & 0.5705 & 0.9310 & $0.049^{*}$ \\
\hline $\mathrm{H} 29 \mathrm{C}$ & 0.6168 & 0.6271 & 0.8587 & $0.049^{*}$ \\
\hline $\mathrm{C} 4$ & $0.3343(2)$ & 0.52907 (14) & $0.8798(3)$ & $0.0284(7)$ \\
\hline $\mathrm{H} 4 \mathrm{~A}$ & 0.3370 & 0.4854 & 0.8837 & $0.034 *$ \\
\hline H4B & 0.3400 & 0.5445 & 0.9460 & $0.034^{*}$ \\
\hline $\mathrm{C} 2$ & $0.4107(2)$ & $0.53720(15)$ & $0.7553(3)$ & $0.0305(7)$ \\
\hline $\mathrm{H} 2 \mathrm{~A}$ & 0.4675 & 0.5565 & 0.7424 & $0.037^{*}$ \\
\hline $\mathrm{H} 2 \mathrm{~B}$ & 0.4163 & 0.4939 & 0.7485 & $0.037^{*}$ \\
\hline $\mathrm{C} 21$ & 0.4188 (3) & $0.55523(19)$ & $0.3467(4)$ & $0.0479(10)$ \\
\hline $\mathrm{H} 21 \mathrm{~A}$ & 0.4791 & 0.5607 & 0.3289 & $0.057^{*}$ \\
\hline H21B & 0.4489 & 0.5465 & 0.4213 & $0.057^{*}$ \\
\hline
\end{tabular}




$\begin{array}{lllll}\text { C24 } & 0.1992(3) & 0.44301(16) & 0.2775(3) & 0.0406(9) \\ \text { H24A } & 0.1483 & 0.4403 & 0.3072 & 0.061^{*} \\ \text { H24B } & 0.2504 & 0.4102 & 0.3028 & 0.061^{*} \\ \text { H24C } & 0.1601 & 0.4409 & 0.2022 & 0.061^{*} \\ \text { C11 } & -0.1538(3) & 0.59608(16) & 0.2335(3) & 0.0401(9) \\ \text { H11A } & -0.0989 & 0.5826 & 0.2134 & 0.060^{*} \\ \text { H11B } & -0.2235 & 0.5977 & 0.1726 & 0.060^{*} \\ \text { H11C } & -0.1579 & 0.5685 & 0.2839 & 0.060^{*} \\ \text { C30 } & 1.0498(3) & 0.5726(2) & 1.0638(4) & 0.0552(1) \\ \text { H30A } & 1.0688 & 0.5512 & 1.0154 & 0.083^{*} \\ \text { H30B } & 1.0319 & 0.5443 & 1.1049 & 0.083^{*} \\ \text { H30C } & 1.1107 & 0.5969 & 1.1095 & 0.083^{*} \\ \text { C9 } & -0.1671(3) & 0.75993(15) & 0.2679(3) & 0.0423(9) \\ \text { H9A } & -0.1890 & 0.7945 & 0.2211 & 0.051^{*} \\ \text { H9B } & -0.2187 & 0.7556 & 0.2975 & 0.051^{*} \\ \text { C10 } & -0.0559(3) & 0.76947(14) & 0.3529(3) & 0.0365(8) \\ \text { H10A } & -0.0069 & 0.7806 & 0.3228 & 0.044^{*} \\ \text { H10B } & -0.0570 & 0.8023 & 0.3976 & 0.044^{*} \\ \text { C22 } & 0.3500(3) & 0.50443(17) & 0.2862(4) & 0.0467(10) \\ \text { H22A } & 0.3258 & 0.5112 & 0.2117 & 0.056^{*} \\ \text { H22B } & 0.3922 & 0.4674 & 0.3055 & 0.056^{*} \\ \text { C23 } & 0.1972(4) & 0.65363(17) & 0.3377(4) & 0.0493(11) \\ \text { H23A } & 0.2429 & 0.6759 & 0.3998 & 0.074^{*} \\ \text { H23B } & 0.1306 & 0.6424 & 0.3407 & 0.074^{*} \\ \text { H23C } & 0.1804 & 0.6783 & 0.2768 & 0.074^{*} \\ \text { O6 } & -0.05183(18) & 0.49339(9) & 0.54105(19) & 0.0250(5) \\ \text { H6 } & -0.077(4) & 0.487(2) & 0.575(4) & 0.051(14)^{*}\end{array}$

Atomic displacement parameters $\left(\AA^{2}\right)$

\begin{tabular}{lllllll}
\hline & $U^{11}$ & $U^{22}$ & $U^{33}$ & $U^{12}$ & $U^{13}$ & $U^{23}$ \\
\hline I1 & $0.04045(13)$ & $0.02150(11)$ & $0.02735(12)$ & $0.00304(8)$ & $0.01372(10)$ & $0.00282(7)$ \\
I2 & $0.03502(13)$ & $0.04120(13)$ & $0.03083(13)$ & $-0.00851(9)$ & $0.01468(10)$ & $0.00502(9)$ \\
A11 & $0.0201(4)$ & $0.0147(4)$ & $0.0245(5)$ & $0.0025(3)$ & $0.0100(4)$ & $0.0015(3)$ \\
O1 & $0.0197(10)$ & $0.0256(11)$ & $0.0251(11)$ & $0.0052(8)$ & $0.0056(9)$ & $0.0025(9)$ \\
O3 & $0.0236(10)$ & $0.0202(10)$ & $0.0275(12)$ & $0.0074(8)$ & $0.0108(9)$ & $0.0007(9)$ \\
N9 & $0.0238(14)$ & $0.0334(15)$ & $0.0315(16)$ & $0.0003(11)$ & $0.0117(12)$ & $0.0067(12)$ \\
N2 & $0.0236(13)$ & $0.0222(13)$ & $0.0254(14)$ & $-0.0010(10)$ & $0.0072(11)$ & $0.0015(11)$ \\
N1 & $0.0178(12)$ & $0.0244(13)$ & $0.0290(14)$ & $0.0001(10)$ & $0.0064(11)$ & $0.0013(11)$ \\
C1 & $0.0244(15)$ & $0.0104(13)$ & $0.0232(16)$ & $-0.0008(11)$ & $0.0037(13)$ & $-0.0004(11)$ \\
O2 & $0.0300(11)$ & $0.0178(10)$ & $0.0282(12)$ & $0.0045(9)$ & $0.0109(10)$ & $0.0059(9)$ \\
N4 & $0.0282(13)$ & $0.0160(12)$ & $0.0200(13)$ & $-0.0007(10)$ & $0.0064(11)$ & $0.0000(10)$ \\
N8 & $0.0378(16)$ & $0.0288(15)$ & $0.0464(18)$ & $-0.0013(12)$ & $0.0292(15)$ & $-0.0037(13)$ \\
C7 & $0.0212(14)$ & $0.0189(14)$ & $0.0223(15)$ & $0.0017(11)$ & $0.0135(13)$ & $0.0023(12)$ \\
O4 & $0.0349(13)$ & $0.0353(13)$ & $0.0415(14)$ & $0.0014(10)$ & $0.0261(11)$ & $0.0045(11)$ \\
C12 & $0.0287(16)$ & $0.0257(16)$ & $0.0249(17)$ & $-0.0043(13)$ & $0.0085(14)$ & $-0.0061(13)$ \\
N5 & $0.0233(13)$ & $0.0242(13)$ & $0.0285(14)$ & $0.0029(11)$ & $0.0099(11)$ & $0.0028(11)$ \\
N6 & $0.0260(13)$ & $0.0184(12)$ & $0.0303(14)$ & $0.0018(10)$ & $0.0145(12)$ & $-0.0012(11)$
\end{tabular}




\begin{tabular}{|c|c|c|c|c|c|c|}
\hline N3 & $0.0251(13)$ & $0.0220(13)$ & $0.0209(13)$ & $-0.0004(10)$ & $0.0053(11)$ & $-0.0014(10)$ \\
\hline $\mathrm{C} 17$ & $0.0308(18)$ & $0.037(2)$ & $0.041(2)$ & $-0.0051(15)$ & $0.0025(16)$ & $-0.0028(17)$ \\
\hline O5 & $0.0497(16)$ & $0.0426(15)$ & $0.0432(16)$ & $0.0126(12)$ & $0.0236(13)$ & $0.0164(12)$ \\
\hline $\mathrm{C} 13$ & $0.0209(14)$ & $0.0201(14)$ & $0.0239(15)$ & $-0.0001(11)$ & $0.0142(13)$ & $0.0033(12)$ \\
\hline $\mathrm{C} 5$ & $0.0252(16)$ & 0.0335 & $0.0347(19)$ & $-0.0034(13)$ & $0.0128(14)$ & $0.0026(14)$ \\
\hline C6 & $0.0355(18)$ & $0.0298(17)$ & $0.0285(18)$ & $0.0015(14)$ & $0.0146(15)$ & $0.0014(14)$ \\
\hline N7 & $0.0409(16)$ & $0.0289(15)$ & $0.0452(18)$ & $-0.0031(12)$ & $0.0304(15)$ & $-0.0029(13)$ \\
\hline N10 & $0.0280(15)$ & $0.055(2)$ & $0.0359(17)$ & $0.0078(14)$ & $0.0106(13)$ & $0.0061(15)$ \\
\hline $\mathrm{C} 20$ & $0.044(2)$ & $0.0361(19)$ & $0.054(2)$ & $-0.0123(16)$ & $0.0319(19)$ & $-0.0053(17)$ \\
\hline $\mathrm{C} 18$ & $0.0308(17)$ & $0.0279(16)$ & $0.0266(17)$ & $-0.0025(13)$ & $0.0105(14)$ & $-0.0029(13)$ \\
\hline $\mathrm{C} 14$ & $0.0291(17)$ & $0.048(2)$ & $0.0304(19)$ & $0.0161(16)$ & $0.0104(15)$ & $0.0080(16)$ \\
\hline $\mathrm{C} 8$ & $0.0393(19)$ & $0.0365(19)$ & $0.0217(17)$ & $-0.0014(15)$ & $0.0041(15)$ & $0.0080(14)$ \\
\hline $\mathrm{C} 15$ & $0.048(2)$ & $0.0300(18)$ & $0.047(2)$ & $0.0204(16)$ & $0.0247(19)$ & $0.0112(16)$ \\
\hline $\mathrm{C} 27$ & $0.044(2)$ & $0.0333(19)$ & $0.039(2)$ & $-0.0115(16)$ & $0.0213(17)$ & $-0.0041(16)$ \\
\hline $\mathrm{C} 26$ & $0.0345(18)$ & $0.0314(17)$ & $0.0288(18)$ & $-0.0045(14)$ & $0.0106(15)$ & $0.0019(14)$ \\
\hline $\mathrm{C} 3$ & $0.0224(15)$ & $0.0243(16)$ & $0.0317(18)$ & $-0.0015(12)$ & $0.0032(14)$ & $0.0001(13)$ \\
\hline C19 & $0.0329(17)$ & $0.0280(16)$ & $0.0267(17)$ & $0.0003(13)$ & $0.0179(14)$ & $0.0006(13)$ \\
\hline $\mathrm{C} 16$ & $0.0379(18)$ & $0.0230(16)$ & $0.045(2)$ & $0.0067(14)$ & $0.0250(17)$ & $-0.0010(14)$ \\
\hline $\mathrm{C} 28$ & $0.0352(19)$ & $0.042(2)$ & $0.050(2)$ & $-0.0098(16)$ & $0.0223(18)$ & $-0.0104(18)$ \\
\hline $\mathrm{C} 25$ & $0.0358(18)$ & $0.039(2)$ & $0.0252(17)$ & $0.0030(15)$ & $0.0150(15)$ & $0.0072(15)$ \\
\hline $\mathrm{C} 29$ & $0.0289(17)$ & $0.0383(18)$ & $0.0306(18)$ & $-0.0023(14)$ & $0.0137(15)$ & $0.0035(15)$ \\
\hline $\mathrm{C} 4$ & $0.0254(16)$ & $0.0258(16)$ & $0.0260(17)$ & $0.0012(13)$ & $0.0043(13)$ & $0.0041(13)$ \\
\hline $\mathrm{C} 2$ & $0.0196(15)$ & $0.0310(17)$ & $0.0352(19)$ & $0.0003(13)$ & $0.0070(14)$ & $0.0000(14)$ \\
\hline $\mathrm{C} 21$ & $0.035(2)$ & $0.052(2)$ & $0.066(3)$ & $-0.0025(17)$ & $0.031(2)$ & $0.002(2)$ \\
\hline $\mathrm{C} 24$ & $0.053(2)$ & $0.0293(18)$ & $0.049(2)$ & $-0.0092(16)$ & $0.031(2)$ & $-0.0088(16)$ \\
\hline C11 & $0.042(2)$ & $0.0320(19)$ & $0.034(2)$ & $-0.0023(15)$ & $0.0050(17)$ & $-0.0084(15)$ \\
\hline $\mathrm{C} 30$ & $0.040(2)$ & $0.069(3)$ & $0.052(3)$ & $0.013(2)$ & $0.016(2)$ & $0.013(2)$ \\
\hline C9 & $0.049(2)$ & $0.0262(18)$ & $0.038(2)$ & $0.0098(16)$ & $0.0061(18)$ & $0.0123(15)$ \\
\hline $\mathrm{C} 10$ & $0.050(2)$ & $0.0159(15)$ & $0.035(2)$ & $0.0000(14)$ & $0.0113(17)$ & 0.0055 (14) \\
\hline $\mathrm{C} 22$ & $0.050(2)$ & $0.038(2)$ & $0.073(3)$ & $0.0029(17)$ & $0.047(2)$ & $-0.001(2)$ \\
\hline $\mathrm{C} 23$ & $0.072(3)$ & $0.0280(19)$ & $0.070(3)$ & $0.0047(18)$ & $0.052(3)$ & $0.0048(18)$ \\
\hline O6 & $0.0320(12)$ & $0.0162(10)$ & $0.0356(13)$ & $0.0016(8)$ & $0.0230(11)$ & $0.0015(9)$ \\
\hline
\end{tabular}

Geometric parameters ( $\left.\AA,{ }^{\circ}\right)$

\begin{tabular}{llll}
\hline Al1-O1 & $1.789(2)$ & $\mathrm{C} 18-\mathrm{H} 18 \mathrm{~B}$ & 0.9800 \\
$\mathrm{~A} 11-\mathrm{O} 2$ & $1.792(2)$ & $\mathrm{C} 18-\mathrm{H} 18 \mathrm{C}$ & 0.9800 \\
$\mathrm{~A} 11-\mathrm{O} 6$ & $1.804(2)$ & $\mathrm{C} 14-\mathrm{C} 15$ & $1.509(5)$ \\
$\mathrm{A} 11-\mathrm{O} 3$ & $1.846(2)$ & $\mathrm{C} 14-\mathrm{H} 14 \mathrm{~A}$ & 0.9900 \\
$\mathrm{~A} 11-\mathrm{O} 6$ & $\mathrm{i}$ & $\mathrm{C} 14-\mathrm{H} 14 \mathrm{~B}$ & 0.9900 \\
$\mathrm{~A} 11-\mathrm{A} 11^{\mathrm{i}}$ & $1.859(2)$ & $\mathrm{C} 8-\mathrm{C} 9$ & $1.507(5)$ \\
$\mathrm{O} 1-\mathrm{C} 1$ & $2.8831(16)$ & $\mathrm{C} 8-\mathrm{H} 8 \mathrm{~A}$ & 0.9900 \\
$\mathrm{O} 3-\mathrm{C} 13$ & $1.290(4)$ & $\mathrm{C} 8-\mathrm{H} 8 \mathrm{~B}$ & 0.9900 \\
$\mathrm{~N} 9-\mathrm{C} 25$ & $1.282(4)$ & $\mathrm{C} 15-\mathrm{C} 16$ & $1.501(5)$ \\
$\mathrm{N} 9-\mathrm{C} 26$ & $1.353(4)$ & $\mathrm{C} 15-\mathrm{H} 15 \mathrm{~A}$ & 0.9900 \\
$\mathrm{~N} 9-\mathrm{C} 29$ & $1.452(4)$ & $\mathrm{C} 15-\mathrm{H} 15 \mathrm{~B}$ & 0.9900 \\
$\mathrm{~N} 2-\mathrm{C} 1$ & $1.452(4)$ & $\mathrm{C} 27-\mathrm{C} 26$ & $1.500(5)$ \\
$\mathrm{N} 2-\mathrm{C} 6$ & $1.324(4)$ & $\mathrm{C} 27-\mathrm{C} 28$ & $1.502(6)$
\end{tabular}




\begin{tabular}{|c|c|c|c|}
\hline $\mathrm{N} 2-\mathrm{C} 4$ & $1.485(4)$ & $\mathrm{C} 27-\mathrm{H} 27 \mathrm{~A}$ & 0.9900 \\
\hline $\mathrm{N} 1-\mathrm{C} 1$ & $1.340(4)$ & $\mathrm{C} 27-\mathrm{H} 27 \mathrm{~B}$ & 0.9900 \\
\hline $\mathrm{N} 1-\mathrm{C} 5$ & $1.458(4)$ & $\mathrm{C} 26-\mathrm{H} 26 \mathrm{~A}$ & 0.9900 \\
\hline $\mathrm{N} 1-\mathrm{C} 2$ & $1.471(4)$ & $\mathrm{C} 26-\mathrm{H} 26 \mathrm{~B}$ & 0.9900 \\
\hline $\mathrm{O} 2-\mathrm{C} 7$ & $1.274(4)$ & $\mathrm{C} 3-\mathrm{C} 4$ & $1.502(5)$ \\
\hline $\mathrm{N} 4-\mathrm{C} 7$ & $1.339(4)$ & $\mathrm{C} 3-\mathrm{C} 2$ & $1.503(5)$ \\
\hline $\mathrm{N} 4-\mathrm{C} 12$ & $1.454(4)$ & $\mathrm{C} 3-\mathrm{H} 3 \mathrm{~A}$ & 0.9900 \\
\hline $\mathrm{N} 4-\mathrm{C} 10$ & $1.471(4)$ & $\mathrm{C} 3-\mathrm{H} 3 \mathrm{~B}$ & 0.9900 \\
\hline $\mathrm{N} 8-\mathrm{C} 19$ & $1.356(4)$ & $\mathrm{C} 16-\mathrm{H} 16 \mathrm{~A}$ & 0.9900 \\
\hline $\mathrm{N} 8-\mathrm{C} 24$ & $1.457(4)$ & C16-H16B & 0.9900 \\
\hline $\mathrm{N} 8-\mathrm{C} 22$ & $1.464(4)$ & $\mathrm{C} 28-\mathrm{H} 28 \mathrm{~A}$ & 0.9900 \\
\hline $\mathrm{C} 7-\mathrm{N} 3$ & $1.330(4)$ & $\mathrm{C} 28-\mathrm{H} 28 \mathrm{~B}$ & 0.9900 \\
\hline $\mathrm{O} 4-\mathrm{C} 19$ & $1.251(4)$ & $\mathrm{C} 29-\mathrm{H} 29 \mathrm{~A}$ & 0.9800 \\
\hline $\mathrm{C} 12-\mathrm{H} 12 \mathrm{~A}$ & 0.9800 & $\mathrm{C} 29-\mathrm{H} 29 \mathrm{~B}$ & 0.9800 \\
\hline $\mathrm{C} 12-\mathrm{H} 12 \mathrm{~B}$ & 0.9800 & $\mathrm{C} 29-\mathrm{H} 29 \mathrm{C}$ & 0.9800 \\
\hline $\mathrm{C} 12-\mathrm{H} 12 \mathrm{C}$ & 0.9800 & $\mathrm{C} 4-\mathrm{H} 4 \mathrm{~A}$ & 0.9900 \\
\hline $\mathrm{N} 5-\mathrm{C} 13$ & $1.336(4)$ & $\mathrm{C} 4-\mathrm{H} 4 \mathrm{~B}$ & 0.9900 \\
\hline $\mathrm{N} 5-\mathrm{C} 17$ & $1.447(4)$ & $\mathrm{C} 2-\mathrm{H} 2 \mathrm{~A}$ & 0.9900 \\
\hline $\mathrm{N} 5-\mathrm{C} 14$ & $1.475(4)$ & $\mathrm{C} 2-\mathrm{H} 2 \mathrm{~B}$ & 0.9900 \\
\hline $\mathrm{N} 6-\mathrm{C} 13$ & $1.332(4)$ & $\mathrm{C} 21-\mathrm{C} 22$ & $1.503(6)$ \\
\hline $\mathrm{N} 6-\mathrm{C} 18$ & $1.465(4)$ & $\mathrm{C} 21-\mathrm{H} 21 \mathrm{~A}$ & 0.9900 \\
\hline N6-C16 & $1.470(4)$ & $\mathrm{C} 21-\mathrm{H} 21 \mathrm{~B}$ & 0.9900 \\
\hline N3-C11 & $1.463(4)$ & $\mathrm{C} 24-\mathrm{H} 24 \mathrm{~A}$ & 0.9800 \\
\hline N3-C8 & $1.464(4)$ & $\mathrm{C} 24-\mathrm{H} 24 \mathrm{~B}$ & 0.9800 \\
\hline C17-H17A & 0.9800 & $\mathrm{C} 24-\mathrm{H} 24 \mathrm{C}$ & 0.9800 \\
\hline C17-H17B & 0.9800 & $\mathrm{C} 11-\mathrm{H} 11 \mathrm{~A}$ & 0.9800 \\
\hline C17-H17C & 0.9800 & C11-H11B & 0.9800 \\
\hline $\mathrm{O} 5-\mathrm{C} 25$ & $1.237(4)$ & $\mathrm{C} 11-\mathrm{H} 11 \mathrm{C}$ & 0.9800 \\
\hline $\mathrm{C} 5-\mathrm{H} 5 \mathrm{~A}$ & 0.9800 & $\mathrm{C} 30-\mathrm{H} 30 \mathrm{~A}$ & 0.9800 \\
\hline C5-H5B & 0.9800 & $\mathrm{C} 30-\mathrm{H} 30 \mathrm{~B}$ & 0.9800 \\
\hline $\mathrm{C} 5-\mathrm{H} 5 \mathrm{C}$ & 0.9800 & $\mathrm{C} 30-\mathrm{H} 30 \mathrm{C}$ & 0.9800 \\
\hline C6- $-\mathrm{H} 6 \mathrm{~A}$ & 0.9800 & $\mathrm{C} 9-\mathrm{C} 10$ & $1.506(5)$ \\
\hline $\mathrm{C} 6-\mathrm{H} 6 \mathrm{~B}$ & 0.9800 & C9-H9A & 0.9900 \\
\hline $\mathrm{C} 6-\mathrm{H} 6 \mathrm{C}$ & 0.9800 & C9-H9B & 0.9900 \\
\hline N7 $-\mathrm{C} 19$ & $1.356(4)$ & $\mathrm{C} 10-\mathrm{H} 10 \mathrm{~A}$ & 0.9900 \\
\hline $\mathrm{N} 7-\mathrm{C} 23$ & $1.454(5)$ & $\mathrm{C} 10-\mathrm{H} 10 \mathrm{~B}$ & 0.9900 \\
\hline $\mathrm{N} 7-\mathrm{C} 20$ & $1.465(4)$ & $\mathrm{C} 22-\mathrm{H} 22 \mathrm{~A}$ & 0.9900 \\
\hline $\mathrm{N} 10-\mathrm{C} 25$ & $1.368(5)$ & $\mathrm{C} 22-\mathrm{H} 22 \mathrm{~B}$ & 0.9900 \\
\hline $\mathrm{N} 10-\mathrm{C} 28$ & $1.444(5)$ & $\mathrm{C} 23-\mathrm{H} 23 \mathrm{~A}$ & 0.9800 \\
\hline $\mathrm{N} 10-\mathrm{C} 30$ & $1.459(5)$ & $\mathrm{C} 23-\mathrm{H} 23 \mathrm{~B}$ & 0.9800 \\
\hline $\mathrm{C} 20-\mathrm{C} 21$ & $1.504(6)$ & $\mathrm{C} 23-\mathrm{H} 23 \mathrm{C}$ & 0.9800 \\
\hline $\mathrm{C} 20-\mathrm{H} 20 \mathrm{~A}$ & 0.9900 & $\mathrm{O} 6-\mathrm{Al1}^{\mathrm{i}}$ & $1.859(2)$ \\
\hline $\mathrm{C} 20-\mathrm{H} 20 \mathrm{~B}$ & 0.9900 & O6-H6 & $0.73(5)$ \\
\hline $\mathrm{C} 18-\mathrm{H} 18 \mathrm{~A}$ & 0.9800 & & \\
\hline $\mathrm{O} 1-\mathrm{A} 11-\mathrm{O} 2$ & $104.24(11)$ & $\mathrm{C} 16-\mathrm{C} 15-\mathrm{H} 15 \mathrm{~B}$ & 109.9 \\
\hline $\mathrm{O} 1-\mathrm{A} 11-\mathrm{O} 6$ & $115.18(11)$ & $\mathrm{C} 14-\mathrm{C} 15-\mathrm{H} 15 \mathrm{~B}$ & 109.9 \\
\hline $\mathrm{O} 2-\mathrm{A} 11-\mathrm{O} 6$ & $139.96(12)$ & $\mathrm{H} 15 \mathrm{~A}-\mathrm{C} 15-\mathrm{H} 15 \mathrm{~B}$ & 108.3 \\
\hline
\end{tabular}




\begin{tabular}{|c|c|c|c|}
\hline $\mathrm{O} 1-\mathrm{A} 11-\mathrm{O} 3$ & $92.39(10)$ & $\mathrm{C} 26-\mathrm{C} 27-\mathrm{C} 28$ & $109.8(3)$ \\
\hline $\mathrm{O} 2-\mathrm{A} 11-\mathrm{O} 3$ & $92.99(10)$ & $\mathrm{C} 26-\mathrm{C} 27-\mathrm{H} 27 \mathrm{~A}$ & 109.7 \\
\hline $\mathrm{O} 6-\mathrm{A} 11-\mathrm{O} 3$ & $92.13(10)$ & $\mathrm{C} 28-\mathrm{C} 27-\mathrm{H} 27 \mathrm{~A}$ & 109.7 \\
\hline $\mathrm{O} 1-\mathrm{A} 11-\mathrm{O}^{\mathrm{i}}$ & $101.27(11)$ & $\mathrm{C} 26-\mathrm{C} 27-\mathrm{H} 27 \mathrm{~B}$ & 109.7 \\
\hline $\mathrm{O} 2-\mathrm{A} 11-\mathrm{O}^{\mathrm{i}}$ & $90.04(11)$ & $\mathrm{C} 28-\mathrm{C} 27-\mathrm{H} 27 \mathrm{~B}$ & 109.7 \\
\hline $\mathrm{O} 6-\mathrm{A} 11-\mathrm{O}^{\mathrm{i}}$ & $76.16(12)$ & $\mathrm{H} 27 \mathrm{~A}-\mathrm{C} 27-\mathrm{H} 27 \mathrm{~B}$ & 108.2 \\
\hline $\mathrm{O} 3-\mathrm{A} 11-\mathrm{O}^{\mathrm{i}}$ & $164.83(11)$ & N9- $\mathrm{C} 26-\mathrm{C} 27$ & $109.9(3)$ \\
\hline $\mathrm{O} 1-\mathrm{A} 11-\mathrm{A} 11^{\mathrm{i}}$ & $113.09(8)$ & $\mathrm{N} 9-\mathrm{C} 26-\mathrm{H} 26 \mathrm{~A}$ & 109.7 \\
\hline $\mathrm{O} 2-\mathrm{A} 11-\mathrm{A} 11^{\mathrm{i}}$ & $118.65(9)$ & $\mathrm{C} 27-\mathrm{C} 26-\mathrm{H} 26 \mathrm{~A}$ & 109.7 \\
\hline $\mathrm{O} 6-\mathrm{A} 11-\mathrm{A} 11^{\mathrm{i}}$ & $38.76(7)$ & $\mathrm{N} 9-\mathrm{C} 26-\mathrm{H} 26 \mathrm{~B}$ & 109.7 \\
\hline $\mathrm{O} 3-\mathrm{A} 11-\mathrm{A} 11^{\mathrm{i}}$ & $130.21(8)$ & $\mathrm{C} 27-\mathrm{C} 26-\mathrm{H} 26 \mathrm{~B}$ & 109.7 \\
\hline $\mathrm{O} 6^{\mathrm{i}}-\mathrm{A} 11-\mathrm{A} 11^{\mathrm{i}}$ & $37.40(7)$ & $\mathrm{H} 26 \mathrm{~A}-\mathrm{C} 26-\mathrm{H} 26 \mathrm{~B}$ & 108.2 \\
\hline $\mathrm{C} 1-\mathrm{O} 1-\mathrm{A} 11$ & $145.4(2)$ & $\mathrm{C} 4-\mathrm{C} 3-\mathrm{C} 2$ & $110.8(3)$ \\
\hline $\mathrm{C} 13-\mathrm{O} 3-\mathrm{A} 11$ & $144.0(2)$ & $\mathrm{C} 4-\mathrm{C} 3-\mathrm{H} 3 \mathrm{~A}$ & 109.5 \\
\hline $\mathrm{C} 25-\mathrm{N} 9-\mathrm{C} 26$ & $123.0(3)$ & $\mathrm{C} 2-\mathrm{C} 3-\mathrm{H} 3 \mathrm{~A}$ & 109.5 \\
\hline $\mathrm{C} 25-\mathrm{N} 9-\mathrm{C} 29$ & $119.2(3)$ & $\mathrm{C} 4-\mathrm{C} 3-\mathrm{H} 3 \mathrm{~B}$ & 109.5 \\
\hline $\mathrm{C} 26-\mathrm{N} 9-\mathrm{C} 29$ & $117.4(3)$ & $\mathrm{C} 2-\mathrm{C} 3-\mathrm{H} 3 \mathrm{~B}$ & 109.5 \\
\hline $\mathrm{C} 1-\mathrm{N} 2-\mathrm{C} 6$ & $121.6(3)$ & $\mathrm{H} 3 \mathrm{~A}-\mathrm{C} 3-\mathrm{H} 3 \mathrm{~B}$ & 108.1 \\
\hline $\mathrm{C} 1-\mathrm{N} 2-\mathrm{C} 4$ & $122.3(3)$ & $\mathrm{O} 4-\mathrm{C} 19-\mathrm{N} 8$ & $120.6(3)$ \\
\hline $\mathrm{C} 6-\mathrm{N} 2-\mathrm{C} 4$ & $116.0(3)$ & $\mathrm{O} 4-\mathrm{C} 19-\mathrm{N} 7$ & $120.9(3)$ \\
\hline $\mathrm{C} 1-\mathrm{N} 1-\mathrm{C} 5$ & $122.3(3)$ & $\mathrm{N} 8-\mathrm{C} 19-\mathrm{N} 7$ & $118.5(3)$ \\
\hline $\mathrm{C} 1-\mathrm{N} 1-\mathrm{C} 2$ & $121.6(3)$ & $\mathrm{N} 6-\mathrm{C} 16-\mathrm{C} 15$ & $108.7(3)$ \\
\hline $\mathrm{C} 5-\mathrm{N} 1-\mathrm{C} 2$ & $116.1(3)$ & $\mathrm{N} 6-\mathrm{C} 16-\mathrm{H} 16 \mathrm{~A}$ & 109.9 \\
\hline $\mathrm{O} 1-\mathrm{C} 1-\mathrm{N} 2$ & $119.2(3)$ & $\mathrm{C} 15-\mathrm{C} 16-\mathrm{H} 16 \mathrm{~A}$ & 109.9 \\
\hline $\mathrm{O} 1-\mathrm{C} 1-\mathrm{N} 1$ & $119.0(3)$ & $\mathrm{N} 6-\mathrm{C} 16-\mathrm{H} 16 \mathrm{~B}$ & 109.9 \\
\hline $\mathrm{N} 2-\mathrm{C} 1-\mathrm{N} 1$ & $121.7(3)$ & $\mathrm{C} 15-\mathrm{C} 16-\mathrm{H} 16 \mathrm{~B}$ & 109.9 \\
\hline $\mathrm{C} 7-\mathrm{O} 2-\mathrm{A} 11$ & $154.7(2)$ & $\mathrm{H} 16 \mathrm{~A}-\mathrm{C} 16-\mathrm{H} 16 \mathrm{~B}$ & 108.3 \\
\hline $\mathrm{C} 7-\mathrm{N} 4-\mathrm{C} 12$ & $120.8(2)$ & $\mathrm{N} 10-\mathrm{C} 28-\mathrm{C} 27$ & $112.2(3)$ \\
\hline $\mathrm{C} 7-\mathrm{N} 4-\mathrm{C} 10$ & $121.9(3)$ & $\mathrm{N} 10-\mathrm{C} 28-\mathrm{H} 28 \mathrm{~A}$ & 109.2 \\
\hline $\mathrm{C} 12-\mathrm{N} 4-\mathrm{C} 10$ & $115.6(2)$ & $\mathrm{C} 27-\mathrm{C} 28-\mathrm{H} 28 \mathrm{~A}$ & 109.2 \\
\hline $\mathrm{C} 19-\mathrm{N} 8-\mathrm{C} 24$ & $119.0(3)$ & $\mathrm{N} 10-\mathrm{C} 28-\mathrm{H} 28 \mathrm{~B}$ & 109.2 \\
\hline $\mathrm{C} 19-\mathrm{N} 8-\mathrm{C} 22$ & $121.8(3)$ & $\mathrm{C} 27-\mathrm{C} 28-\mathrm{H} 28 \mathrm{~B}$ & 109.2 \\
\hline $\mathrm{C} 24-\mathrm{N} 8-\mathrm{C} 22$ & $115.7(3)$ & $\mathrm{H} 28 \mathrm{~A}-\mathrm{C} 28-\mathrm{H} 28 \mathrm{~B}$ & 107.9 \\
\hline $\mathrm{O} 2-\mathrm{C} 7-\mathrm{N} 3$ & $118.8(3)$ & $\mathrm{O} 5-\mathrm{C} 25-\mathrm{N} 9$ & $121.0(3)$ \\
\hline $\mathrm{O} 2-\mathrm{C} 7-\mathrm{N} 4$ & $120.3(3)$ & $\mathrm{O} 5-\mathrm{C} 25-\mathrm{N} 10$ & $122.1(3)$ \\
\hline $\mathrm{N} 3-\mathrm{C} 7-\mathrm{N} 4$ & $121.0(3)$ & $\mathrm{N} 9-\mathrm{C} 25-\mathrm{N} 10$ & $116.9(3)$ \\
\hline $\mathrm{N} 4-\mathrm{C} 12-\mathrm{H} 12 \mathrm{~A}$ & 109.5 & N9- $\mathrm{C} 29-\mathrm{H} 29 \mathrm{~A}$ & 109.5 \\
\hline $\mathrm{N} 4-\mathrm{C} 12-\mathrm{H} 12 \mathrm{~B}$ & 109.5 & N9- $\mathrm{C} 29-\mathrm{H} 29 \mathrm{~B}$ & 109.5 \\
\hline $\mathrm{H} 12 \mathrm{~A}-\mathrm{C} 12-\mathrm{H} 12 \mathrm{~B}$ & 109.5 & $\mathrm{H} 29 \mathrm{~A}-\mathrm{C} 29-\mathrm{H} 29 \mathrm{~B}$ & 109.5 \\
\hline $\mathrm{N} 4-\mathrm{C} 12-\mathrm{H} 12 \mathrm{C}$ & 109.5 & N9- $\mathrm{C} 29-\mathrm{H} 29 \mathrm{C}$ & 109.5 \\
\hline $\mathrm{H} 12 \mathrm{~A}-\mathrm{C} 12-\mathrm{H} 12 \mathrm{C}$ & 109.5 & $\mathrm{H} 29 \mathrm{~A}-\mathrm{C} 29-\mathrm{H} 29 \mathrm{C}$ & 109.5 \\
\hline $\mathrm{H} 12 \mathrm{~B}-\mathrm{C} 12-\mathrm{H} 12 \mathrm{C}$ & 109.5 & $\mathrm{H} 29 \mathrm{~B}-\mathrm{C} 29-\mathrm{H} 29 \mathrm{C}$ & 109.5 \\
\hline $\mathrm{C} 13-\mathrm{N} 5-\mathrm{C} 17$ & $120.3(3)$ & $\mathrm{N} 2-\mathrm{C} 4-\mathrm{C} 3$ & $110.1(3)$ \\
\hline $\mathrm{C} 13-\mathrm{N} 5-\mathrm{C} 14$ & $122.9(3)$ & $\mathrm{N} 2-\mathrm{C} 4-\mathrm{H} 4 \mathrm{~A}$ & 109.6 \\
\hline $\mathrm{C} 17-\mathrm{N} 5-\mathrm{C} 14$ & $115.2(3)$ & $\mathrm{C} 3-\mathrm{C} 4-\mathrm{H} 4 \mathrm{~A}$ & 109.6 \\
\hline $\mathrm{C} 13-\mathrm{N} 6-\mathrm{C} 18$ & $120.9(3)$ & $\mathrm{N} 2-\mathrm{C} 4-\mathrm{H} 4 \mathrm{~B}$ & 109.6 \\
\hline $\mathrm{C} 13-\mathrm{N} 6-\mathrm{C} 16$ & $121.1(3)$ & $\mathrm{C} 3-\mathrm{C} 4-\mathrm{H} 4 \mathrm{~B}$ & 109.6 \\
\hline $\mathrm{C} 18-\mathrm{N} 6-\mathrm{C} 16$ & $117.6(3)$ & $\mathrm{H} 4 \mathrm{~A}-\mathrm{C} 4-\mathrm{H} 4 \mathrm{~B}$ & 108.1 \\
\hline
\end{tabular}




\begin{tabular}{|c|c|}
\hline $\mathrm{C} 7-\mathrm{N} 3-\mathrm{C} 11$ & $120.4(3)$ \\
\hline $\mathrm{C} 7-\mathrm{N} 3-\mathrm{C} 8$ & $122.3(3)$ \\
\hline $\mathrm{C} 11-\mathrm{N} 3-\mathrm{C} 8$ & $116.2(3)$ \\
\hline $\mathrm{N} 5-\mathrm{C} 17-\mathrm{H} 17 \mathrm{~A}$ & 109.5 \\
\hline N5-C17-H17B & 109.5 \\
\hline $\mathrm{H} 17 \mathrm{~A}-\mathrm{C} 17-\mathrm{H} 17 \mathrm{~B}$ & 109.5 \\
\hline $\mathrm{N} 5-\mathrm{C} 17-\mathrm{H} 17 \mathrm{C}$ & 109.5 \\
\hline $\mathrm{H} 17 \mathrm{~A}-\mathrm{C} 17-\mathrm{H} 17 \mathrm{C}$ & 109.5 \\
\hline $\mathrm{H} 17 \mathrm{~B}-\mathrm{C} 17-\mathrm{H} 17 \mathrm{C}$ & 109.5 \\
\hline $\mathrm{O} 3-\mathrm{C} 13-\mathrm{N} 6$ & $119.3(3)$ \\
\hline $\mathrm{O} 3-\mathrm{C} 13-\mathrm{N} 5$ & $120.2(3)$ \\
\hline $\mathrm{N} 6-\mathrm{C} 13-\mathrm{N} 5$ & $120.5(3)$ \\
\hline $\mathrm{N} 1-\mathrm{C} 5-\mathrm{H} 5 \mathrm{~A}$ & 109.5 \\
\hline $\mathrm{N} 1-\mathrm{C} 5-\mathrm{H} 5 \mathrm{~B}$ & 109.5 \\
\hline $\mathrm{H} 5 \mathrm{~A}-\mathrm{C} 5-\mathrm{H} 5 \mathrm{~B}$ & 109.5 \\
\hline $\mathrm{N} 1-\mathrm{C} 5-\mathrm{H} 5 \mathrm{C}$ & 109.5 \\
\hline $\mathrm{H} 5 \mathrm{~A}-\mathrm{C} 5-\mathrm{H} 5 \mathrm{C}$ & 109.5 \\
\hline $\mathrm{H} 5 \mathrm{~B}-\mathrm{C} 5-\mathrm{H} 5 \mathrm{C}$ & 109.5 \\
\hline $\mathrm{N} 2-\mathrm{C} 6-\mathrm{H} 6 \mathrm{~A}$ & 109.5 \\
\hline $\mathrm{N} 2-\mathrm{C} 6-\mathrm{H} 6 \mathrm{~B}$ & 109.5 \\
\hline $\mathrm{H} 6 \mathrm{~A}-\mathrm{C} 6-\mathrm{H} 6 \mathrm{~B}$ & 109.5 \\
\hline $\mathrm{N} 2-\mathrm{C} 6-\mathrm{H} 6 \mathrm{C}$ & 109.5 \\
\hline $\mathrm{H} 6 \mathrm{~A}-\mathrm{C} 6-\mathrm{H} 6 \mathrm{C}$ & 109.5 \\
\hline $\mathrm{H} 6 \mathrm{~B}-\mathrm{C} 6-\mathrm{H} 6 \mathrm{C}$ & 109.5 \\
\hline $\mathrm{C} 19-\mathrm{N} 7-\mathrm{C} 23$ & $119.9(3)$ \\
\hline $\mathrm{C} 19-\mathrm{N} 7-\mathrm{C} 20$ & $124.1(3)$ \\
\hline $\mathrm{C} 23-\mathrm{N} 7-\mathrm{C} 20$ & $115.4(3)$ \\
\hline $\mathrm{C} 25-\mathrm{N} 10-\mathrm{C} 28$ & $124.9(3)$ \\
\hline $\mathrm{C} 25-\mathrm{N} 10-\mathrm{C} 30$ & $119.2(3)$ \\
\hline $\mathrm{C} 28-\mathrm{N} 10-\mathrm{C} 30$ & $115.9(3)$ \\
\hline $\mathrm{N} 7-\mathrm{C} 20-\mathrm{C} 21$ & $110.6(3)$ \\
\hline $\mathrm{N} 7-\mathrm{C} 20-\mathrm{H} 20 \mathrm{~A}$ & 109.5 \\
\hline $\mathrm{C} 21-\mathrm{C} 20-\mathrm{H} 20 \mathrm{~A}$ & 109.5 \\
\hline $\mathrm{N} 7-\mathrm{C} 20-\mathrm{H} 20 \mathrm{~B}$ & 109.5 \\
\hline $\mathrm{C} 21-\mathrm{C} 20-\mathrm{H} 20 \mathrm{~B}$ & 109.5 \\
\hline $\mathrm{H} 20 \mathrm{~A}-\mathrm{C} 20-\mathrm{H} 20 \mathrm{~B}$ & 108.1 \\
\hline $\mathrm{N} 6-\mathrm{C} 18-\mathrm{H} 18 \mathrm{~A}$ & 109.5 \\
\hline N6-C18-H18B & 109.5 \\
\hline $\mathrm{H} 18 \mathrm{~A}-\mathrm{C} 18-\mathrm{H} 18 \mathrm{~B}$ & 109.5 \\
\hline $\mathrm{N} 6-\mathrm{C} 18-\mathrm{H} 18 \mathrm{C}$ & 109.5 \\
\hline $\mathrm{H} 18 \mathrm{~A}-\mathrm{C} 18-\mathrm{H} 18 \mathrm{C}$ & 109.5 \\
\hline $\mathrm{H} 18 \mathrm{~B}-\mathrm{C} 18-\mathrm{H} 18 \mathrm{C}$ & 109.5 \\
\hline $\mathrm{N} 5-\mathrm{C} 14-\mathrm{C} 15$ & $110.9(3)$ \\
\hline $\mathrm{N} 5-\mathrm{C} 14-\mathrm{H} 14 \mathrm{~A}$ & 109.5 \\
\hline $\mathrm{C} 15-\mathrm{C} 14-\mathrm{H} 14 \mathrm{~A}$ & 109.5 \\
\hline $\mathrm{N} 5-\mathrm{C} 14-\mathrm{H} 14 \mathrm{~B}$ & 109.5 \\
\hline $\mathrm{C} 15-\mathrm{C} 14-\mathrm{H} 14 \mathrm{~B}$ & 109.5 \\
\hline $\mathrm{H} 14 \mathrm{~A}-\mathrm{C} 14-\mathrm{H} 14 \mathrm{~B}$ & 108.0 \\
\hline
\end{tabular}

\begin{tabular}{|c|c|}
\hline $\mathrm{N} 1-\mathrm{C} 2-\mathrm{C} 3$ & $110.1(3)$ \\
\hline $\mathrm{N} 1-\mathrm{C} 2-\mathrm{H} 2 \mathrm{~A}$ & 109.6 \\
\hline $\mathrm{C} 3-\mathrm{C} 2-\mathrm{H} 2 \mathrm{~A}$ & 109.6 \\
\hline $\mathrm{N} 1-\mathrm{C} 2-\mathrm{H} 2 \mathrm{~B}$ & 109.6 \\
\hline $\mathrm{C} 3-\mathrm{C} 2-\mathrm{H} 2 \mathrm{~B}$ & 109.6 \\
\hline $\mathrm{H} 2 \mathrm{~A}-\mathrm{C} 2-\mathrm{H} 2 \mathrm{~B}$ & 108.2 \\
\hline $\mathrm{C} 22-\mathrm{C} 21-\mathrm{C} 20$ & $109.9(3)$ \\
\hline $\mathrm{C} 22-\mathrm{C} 21-\mathrm{H} 21 \mathrm{~A}$ & 109.7 \\
\hline $\mathrm{C} 20-\mathrm{C} 21-\mathrm{H} 21 \mathrm{~A}$ & 109.7 \\
\hline $\mathrm{C} 22-\mathrm{C} 21-\mathrm{H} 21 \mathrm{~B}$ & 109.7 \\
\hline $\mathrm{C} 20-\mathrm{C} 21-\mathrm{H} 21 \mathrm{~B}$ & 109.7 \\
\hline $\mathrm{H} 21 \mathrm{~A}-\mathrm{C} 21-\mathrm{H} 21 \mathrm{~B}$ & 108.2 \\
\hline $\mathrm{N} 8-\mathrm{C} 24-\mathrm{H} 24 \mathrm{~A}$ & 109.5 \\
\hline $\mathrm{N} 8-\mathrm{C} 24-\mathrm{H} 24 \mathrm{~B}$ & 109.5 \\
\hline $\mathrm{H} 24 \mathrm{~A}-\mathrm{C} 24-\mathrm{H} 24 \mathrm{~B}$ & 109.5 \\
\hline $\mathrm{N} 8-\mathrm{C} 24-\mathrm{H} 24 \mathrm{C}$ & 109.5 \\
\hline $\mathrm{H} 24 \mathrm{~A}-\mathrm{C} 24-\mathrm{H} 24 \mathrm{C}$ & 109.5 \\
\hline $\mathrm{H} 24 \mathrm{~B}-\mathrm{C} 24-\mathrm{H} 24 \mathrm{C}$ & 109.5 \\
\hline $\mathrm{N} 3-\mathrm{C} 11-\mathrm{H} 11 \mathrm{~A}$ & 109.5 \\
\hline $\mathrm{N} 3-\mathrm{C} 11-\mathrm{H} 11 \mathrm{~B}$ & 109.5 \\
\hline $\mathrm{H} 11 \mathrm{~A}-\mathrm{C} 11-\mathrm{H} 11 \mathrm{~B}$ & 109.5 \\
\hline $\mathrm{N} 3-\mathrm{C} 11-\mathrm{H} 11 \mathrm{C}$ & 109.5 \\
\hline $\mathrm{H} 11 \mathrm{~A}-\mathrm{C} 11-\mathrm{H} 11 \mathrm{C}$ & 109.5 \\
\hline $\mathrm{H} 11 \mathrm{~B}-\mathrm{C} 11-\mathrm{H} 11 \mathrm{C}$ & 109.5 \\
\hline $\mathrm{N} 10-\mathrm{C} 30-\mathrm{H} 30 \mathrm{~A}$ & 109.5 \\
\hline $\mathrm{N} 10-\mathrm{C} 30-\mathrm{H} 30 \mathrm{~B}$ & 109.5 \\
\hline $\mathrm{H} 30 \mathrm{~A}-\mathrm{C} 30-\mathrm{H} 30 \mathrm{~B}$ & 109.5 \\
\hline $\mathrm{N} 10-\mathrm{C} 30-\mathrm{H} 30 \mathrm{C}$ & 109.5 \\
\hline $\mathrm{H} 30 \mathrm{~A}-\mathrm{C} 30-\mathrm{H} 30 \mathrm{C}$ & 109.5 \\
\hline $\mathrm{H} 30 \mathrm{~B}-\mathrm{C} 30-\mathrm{H} 30 \mathrm{C}$ & 109.5 \\
\hline $\mathrm{C} 10-\mathrm{C} 9-\mathrm{C} 8$ & $109.4(3)$ \\
\hline $\mathrm{C} 10-\mathrm{C} 9-\mathrm{H} 9 \mathrm{~A}$ & 109.8 \\
\hline $\mathrm{C} 8-\mathrm{C} 9-\mathrm{H} 9 \mathrm{~A}$ & 109.8 \\
\hline $\mathrm{C} 10-\mathrm{C} 9-\mathrm{H} 9 \mathrm{~B}$ & 109.8 \\
\hline $\mathrm{C} 8-\mathrm{C} 9-\mathrm{H} 9 \mathrm{~B}$ & 109.8 \\
\hline $\mathrm{H} 9 \mathrm{~A}-\mathrm{C} 9-\mathrm{H} 9 \mathrm{~B}$ & 108.2 \\
\hline $\mathrm{N} 4-\mathrm{C} 10-\mathrm{C} 9$ & $110.6(3)$ \\
\hline $\mathrm{N} 4-\mathrm{C} 10-\mathrm{H} 10 \mathrm{~A}$ & 109.5 \\
\hline $\mathrm{C} 9-\mathrm{C} 10-\mathrm{H} 10 \mathrm{~A}$ & 109.5 \\
\hline $\mathrm{N} 4-\mathrm{C} 10-\mathrm{H} 10 \mathrm{~B}$ & 109.5 \\
\hline $\mathrm{C} 9-\mathrm{C} 10-\mathrm{H} 10 \mathrm{~B}$ & 109.5 \\
\hline $\mathrm{H} 10 \mathrm{~A}-\mathrm{C} 10-\mathrm{H} 10 \mathrm{~B}$ & 108.1 \\
\hline $\mathrm{N} 8-\mathrm{C} 22-\mathrm{C} 21$ & $109.6(3)$ \\
\hline $\mathrm{N} 8-\mathrm{C} 22-\mathrm{H} 22 \mathrm{~A}$ & 109.7 \\
\hline $\mathrm{C} 21-\mathrm{C} 22-\mathrm{H} 22 \mathrm{~A}$ & 109.7 \\
\hline $\mathrm{N} 8-\mathrm{C} 22-\mathrm{H} 22 \mathrm{~B}$ & 109.7 \\
\hline $\mathrm{C} 21-\mathrm{C} 22-\mathrm{H} 22 \mathrm{~B}$ & 109.7 \\
\hline $\mathrm{H} 22 \mathrm{~A}-\mathrm{C} 22-\mathrm{H} 22 \mathrm{~B}$ & 108.2 \\
\hline
\end{tabular}




\begin{tabular}{|c|c|c|c|}
\hline $\mathrm{N} 3-\mathrm{C} 8-\mathrm{C} 9$ & $109.7(3)$ & $\mathrm{N} 7-\mathrm{C} 23-\mathrm{H} 23 \mathrm{~A}$ & 109.5 \\
\hline $\mathrm{N} 3-\mathrm{C} 8-\mathrm{H} 8 \mathrm{~A}$ & 109.7 & $\mathrm{~N} 7-\mathrm{C} 23-\mathrm{H} 23 \mathrm{~B}$ & 109.5 \\
\hline $\mathrm{C} 9-\mathrm{C} 8-\mathrm{H} 8 \mathrm{~A}$ & 109.7 & $\mathrm{H} 23 \mathrm{~A}-\mathrm{C} 23-\mathrm{H} 23 \mathrm{~B}$ & 109.5 \\
\hline $\mathrm{N} 3-\mathrm{C} 8-\mathrm{H} 8 \mathrm{~B}$ & 109.7 & $\mathrm{~N} 7-\mathrm{C} 23-\mathrm{H} 23 \mathrm{C}$ & 109.5 \\
\hline $\mathrm{C} 9-\mathrm{C} 8-\mathrm{H} 8 \mathrm{~B}$ & 109.7 & $\mathrm{H} 23 \mathrm{~A}-\mathrm{C} 23-\mathrm{H} 23 \mathrm{C}$ & 109.5 \\
\hline $\mathrm{H} 8 \mathrm{~A}-\mathrm{C} 8-\mathrm{H} 8 \mathrm{~B}$ & 108.2 & $\mathrm{H} 23 \mathrm{~B}-\mathrm{C} 23-\mathrm{H} 23 \mathrm{C}$ & 109.5 \\
\hline $\mathrm{C} 16-\mathrm{C} 15-\mathrm{C} 14$ & $109.0(3)$ & $\mathrm{A} 11-\mathrm{O} 6-\mathrm{A} 11^{\mathrm{i}}$ & $103.84(12)$ \\
\hline $\mathrm{C} 16-\mathrm{C} 15-\mathrm{H} 15 \mathrm{~A}$ & 109.9 & $\mathrm{~A} 11-\mathrm{O} 6-\mathrm{H} 6$ & $128(4)$ \\
\hline $\mathrm{C} 14-\mathrm{C} 15-\mathrm{H} 15 \mathrm{~A}$ & 109.9 & $\mathrm{~A} 11^{\mathrm{i}}-\mathrm{O} 6-\mathrm{H} 6$ & $127(4)$ \\
\hline $\mathrm{O} 2-\mathrm{A} 11-\mathrm{O} 1-\mathrm{C} 1$ & $-133.5(4)$ & $\mathrm{C} 17-\mathrm{N} 5-\mathrm{C} 14-\mathrm{C} 15$ & $-174.3(3)$ \\
\hline $\mathrm{O} 6-\mathrm{A} 11-\mathrm{O} 1-\mathrm{C} 1$ & $39.4(4)$ & $\mathrm{C} 7-\mathrm{N} 3-\mathrm{C} 8-\mathrm{C} 9$ & $-32.0(4)$ \\
\hline $\mathrm{O} 3-\mathrm{A} 11-\mathrm{O} 1-\mathrm{C} 1$ & $132.8(4)$ & $\mathrm{C} 11-\mathrm{N} 3-\mathrm{C} 8-\mathrm{C} 9$ & $160.2(3)$ \\
\hline $\mathrm{O} 6 \mathrm{i}-\mathrm{A} 11-\mathrm{O} 1-\mathrm{C} 1$ & $-40.5(4)$ & $\mathrm{N} 5-\mathrm{C} 14-\mathrm{C} 15-\mathrm{C} 16$ & $-49.1(4)$ \\
\hline $\mathrm{A} 11^{\mathrm{i}}-\mathrm{A} 11-\mathrm{O} 1-\mathrm{C} 1$ & $-3.2(4)$ & $\mathrm{C} 25-\mathrm{N} 9-\mathrm{C} 26-\mathrm{C} 27$ & $36.6(4)$ \\
\hline $\mathrm{O} 1-\mathrm{A} 11-\mathrm{O} 3-\mathrm{C} 13$ & $117.8(3)$ & $\mathrm{C} 29-\mathrm{N} 9-\mathrm{C} 26-\mathrm{C} 27$ & $-151.0(3)$ \\
\hline $\mathrm{O} 2-\mathrm{A} 11-\mathrm{O} 3-\mathrm{C} 13$ & $13.4(4)$ & $\mathrm{C} 28-\mathrm{C} 27-\mathrm{C} 26-\mathrm{N} 9$ & $-54.5(4)$ \\
\hline $\mathrm{O} 6-\mathrm{A} 11-\mathrm{O} 3-\mathrm{C} 13$ & $-126.8(3)$ & $\mathrm{C} 24-\mathrm{N} 8-\mathrm{C} 19-\mathrm{O} 4$ & $12.2(5)$ \\
\hline $\mathrm{O} 6-\mathrm{A} 11-\mathrm{O} 3-\mathrm{C} 13$ & $-87.8(5)$ & $\mathrm{C} 22-\mathrm{N} 8-\mathrm{C} 19-\mathrm{O} 4$ & $170.1(3)$ \\
\hline $\mathrm{A} 11 \mathrm{i}-\mathrm{A} 11-\mathrm{O} 3-\mathrm{C} 13$ & $-118.8(3)$ & $\mathrm{C} 24-\mathrm{N} 8-\mathrm{C} 19-\mathrm{N} 7$ & $-169.3(3)$ \\
\hline $\mathrm{A} 11-\mathrm{O} 1-\mathrm{C} 1-\mathrm{N} 2$ & $-94.1(4)$ & $\mathrm{C} 22-\mathrm{N} 8-\mathrm{C} 19-\mathrm{N} 7$ & $-11.4(5)$ \\
\hline $\mathrm{A} 11-\mathrm{O} 1-\mathrm{C} 1-\mathrm{N} 1$ & $88.0(4)$ & $\mathrm{C} 23-\mathrm{N} 7-\mathrm{C} 19-\mathrm{O} 4$ & $-6.2(5)$ \\
\hline $\mathrm{C} 6-\mathrm{N} 2-\mathrm{C} 1-\mathrm{O} 1$ & $-0.1(4)$ & $\mathrm{C} 20-\mathrm{N} 7-\mathrm{C} 19-\mathrm{O} 4$ & $-176.9(3)$ \\
\hline $\mathrm{C} 4-\mathrm{N} 2-\mathrm{C} 1-\mathrm{O} 1$ & $-176.1(3)$ & $\mathrm{C} 23-\mathrm{N} 7-\mathrm{C} 19-\mathrm{N} 8$ & $175.3(3)$ \\
\hline $\mathrm{C} 6-\mathrm{N} 2-\mathrm{C} 1-\mathrm{N} 1$ & $177.7(3)$ & $\mathrm{C} 20-\mathrm{N} 7-\mathrm{C} 19-\mathrm{N} 8$ & $4.6(5)$ \\
\hline $\mathrm{C} 4-\mathrm{N} 2-\mathrm{C} 1-\mathrm{N} 1$ & $1.7(4)$ & $\mathrm{C} 13-\mathrm{N} 6-\mathrm{C} 16-\mathrm{C} 15$ & $-39.2(4)$ \\
\hline $\mathrm{C} 5-\mathrm{N} 1-\mathrm{C} 1-\mathrm{O} 1$ & $-2.8(4)$ & $\mathrm{C} 18-\mathrm{N} 6-\mathrm{C} 16-\mathrm{C} 15$ & $147.6(3)$ \\
\hline $\mathrm{C} 2-\mathrm{N} 1-\mathrm{C} 1-\mathrm{O} 1$ & $178.6(3)$ & $\mathrm{C} 14-\mathrm{C} 15-\mathrm{C} 16-\mathrm{N} 6$ & $57.8(4)$ \\
\hline $\mathrm{C} 5-\mathrm{N} 1-\mathrm{C} 1-\mathrm{N} 2$ & $179.4(3)$ & $\mathrm{C} 25-\mathrm{N} 10-\mathrm{C} 28-\mathrm{C} 27$ & $-15.1(5)$ \\
\hline $\mathrm{C} 2-\mathrm{N} 1-\mathrm{C} 1-\mathrm{N} 2$ & $0.8(4)$ & $\mathrm{C} 30-\mathrm{N} 10-\mathrm{C} 28-\mathrm{C} 27$ & $165.3(3)$ \\
\hline $\mathrm{O} 1-\mathrm{A} 11-\mathrm{O} 2-\mathrm{C} 7$ & $-104.2(5)$ & $\mathrm{C} 26-\mathrm{C} 27-\mathrm{C} 28-\mathrm{N} 10$ & $44.8(4)$ \\
\hline $\mathrm{O} 6-\mathrm{A} 11-\mathrm{O} 2-\mathrm{C} 7$ & $86.0(5)$ & $\mathrm{C} 26-\mathrm{N} 9-\mathrm{C} 25-\mathrm{O} 5$ & $174.5(3)$ \\
\hline $\mathrm{O} 3-\mathrm{A} 11-\mathrm{O} 2-\mathrm{C} 7$ & $-10.9(5)$ & $\mathrm{C} 29-\mathrm{N} 9-\mathrm{C} 25-\mathrm{O} 5$ & $2.3(5)$ \\
\hline $\mathrm{O} 6 \mathrm{i}-\mathrm{A} 11-\mathrm{O} 2-\mathrm{C} 7$ & $154.2(5)$ & $\mathrm{C} 26-\mathrm{N} 9-\mathrm{C} 25-\mathrm{N} 10$ & $-5.6(5)$ \\
\hline $\mathrm{A} 11-\mathrm{A} 11-\mathrm{O} 2-\mathrm{C} 7$ & $129.0(5)$ & $\mathrm{C} 29-\mathrm{N} 9-\mathrm{C} 25-\mathrm{N} 10$ & $-177.9(3)$ \\
\hline $\mathrm{A} 11-\mathrm{O} 2-\mathrm{C} 7-\mathrm{N} 3$ & $-101.8(5)$ & $\mathrm{C} 28-\mathrm{N} 10-\mathrm{C} 25-\mathrm{O} 5$ & $173.8(4)$ \\
\hline $\mathrm{A} 11-\mathrm{O} 2-\mathrm{C} 7-\mathrm{N} 4$ & $79.3(6)$ & $\mathrm{C} 30-\mathrm{N} 10-\mathrm{C} 25-\mathrm{O} 5$ & $-6.6(6)$ \\
\hline $\mathrm{C} 12-\mathrm{N} 4-\mathrm{C} 7-\mathrm{O} 2$ & $11.5(4)$ & $\mathrm{C} 28-\mathrm{N} 10-\mathrm{C} 25-\mathrm{N} 9$ & $-6.1(5)$ \\
\hline $\mathrm{C} 10-\mathrm{N} 4-\mathrm{C} 7-\mathrm{O} 2$ & $176.0(3)$ & $\mathrm{C} 30-\mathrm{N} 10-\mathrm{C} 25-\mathrm{N} 9$ & $173.6(3)$ \\
\hline $\mathrm{C} 12-\mathrm{N} 4-\mathrm{C} 7-\mathrm{N} 3$ & $-167.3(3)$ & $\mathrm{C} 1-\mathrm{N} 2-\mathrm{C} 4-\mathrm{C} 3$ & $23.8(4)$ \\
\hline $\mathrm{C} 10-\mathrm{N} 4-\mathrm{C} 7-\mathrm{N} 3$ & $-2.8(4)$ & $\mathrm{C} 6-\mathrm{N} 2-\mathrm{C} 4-\mathrm{C} 3$ & $-152.4(3)$ \\
\hline $\mathrm{O} 2-\mathrm{C} 7-\mathrm{N} 3-\mathrm{C} 11$ & $-6.0(4)$ & $\mathrm{C} 2-\mathrm{C} 3-\mathrm{C} 4-\mathrm{N} 2$ & $-50.2(4)$ \\
\hline $\mathrm{N} 4-\mathrm{C} 7-\mathrm{N} 3-\mathrm{C} 11$ & $172.9(3)$ & $\mathrm{C} 1-\mathrm{N} 1-\mathrm{C} 2-\mathrm{C} 3$ & $-28.5(4)$ \\
\hline $\mathrm{O} 2-\mathrm{C} 7-\mathrm{N} 3-\mathrm{C} 8$ & $-173.3(3)$ & $\mathrm{C} 5-\mathrm{N} 1-\mathrm{C} 2-\mathrm{C} 3$ & $152.8(3)$ \\
\hline $\mathrm{N} 4-\mathrm{C} 7-\mathrm{N} 3-\mathrm{C} 8$ & $5.6(4)$ & $\mathrm{C} 4-\mathrm{C} 3-\mathrm{C} 2-\mathrm{N} 1$ & $52.6(4)$ \\
\hline $\mathrm{A} 11-\mathrm{O} 3-\mathrm{C} 13-\mathrm{N} 6$ & $-117.6(3)$ & $\mathrm{N} 7-\mathrm{C} 20-\mathrm{C} 21-\mathrm{C} 22$ & $49.4(5)$ \\
\hline $\mathrm{A} 11-\mathrm{O} 3-\mathrm{C} 13-\mathrm{N} 5$ & $63.2(4)$ & $\mathrm{N} 3-\mathrm{C} 8-\mathrm{C} 9-\mathrm{C} 10$ & $53.8(4)$ \\
\hline $\mathrm{C} 18-\mathrm{N} 6-\mathrm{C} 13-\mathrm{O} 3$ & $2.6(4)$ & $\mathrm{C} 7-\mathrm{N} 4-\mathrm{C} 10-\mathrm{C} 9$ & $27.0(4)$ \\
\hline
\end{tabular}




$\begin{array}{llll}\mathrm{C} 16-\mathrm{N} 6-\mathrm{C} 13-\mathrm{O} 3 & -170.4(3) & \mathrm{C} 12-\mathrm{N} 4-\mathrm{C} 10-\mathrm{C} 9 & -167.7(3) \\ \mathrm{C} 18-\mathrm{N} 6-\mathrm{C} 13-\mathrm{N} 5 & -178.1(3) & \mathrm{C} 8-\mathrm{C} 9-\mathrm{C} 10-\mathrm{N} 4 & -51.7(4) \\ \mathrm{C} 16-\mathrm{N} 6-\mathrm{C} 13-\mathrm{N} 5 & 8.9(4) & \mathrm{C} 19-\mathrm{N} 8-\mathrm{C} 22-\mathrm{C} 21 & 37.6(5) \\ \mathrm{C} 17-\mathrm{N} 5-\mathrm{C} 13-\mathrm{O} 3 & 15.7(4) & \mathrm{C} 24-\mathrm{N} 8-\mathrm{C} 22-\mathrm{C} 21 & -163.8(3) \\ \mathrm{C} 14-\mathrm{N} 5-\mathrm{C} 13-\mathrm{O} 3 & -179.4(3) & \mathrm{C} 20-\mathrm{C} 21-\mathrm{C} 22-\mathrm{N} 8 & -55.5(5) \\ \mathrm{C} 17-\mathrm{N} 5-\mathrm{C} 13-\mathrm{N} 6 & -163.6(3) & \mathrm{O} 1-\mathrm{A} 11-\mathrm{O}-\mathrm{A} 11^{\mathrm{i}} & -96.12(13) \\ \mathrm{C} 14-\mathrm{N} 5-\mathrm{C} 13-\mathrm{N} 6 & 1.3(4) & \mathrm{O} 2-\mathrm{A} 11-\mathrm{O} 6-\mathrm{A} 11^{\mathrm{i}} & 73.02(19) \\ \mathrm{C} 19-\mathrm{N} 7-\mathrm{C} 20-\mathrm{C} 21 & -24.8(5) & \mathrm{O} 3-\mathrm{A} 11-\mathrm{O}-\mathrm{A} 11^{\mathrm{i}} & 170.23(12) \\ \mathrm{C} 23-\mathrm{N} 7-\mathrm{C} 20-\mathrm{C} 21 & 164.2(4) & \mathrm{O} 6-\mathrm{A} 11-\mathrm{O} 6-\mathrm{A} 11^{\mathrm{i}} & 0.000(1) \\ \mathrm{C} 13-\mathrm{N} 5-\mathrm{C} 14-\mathrm{C} 15 & 20.1(4) & & \end{array}$

Symmetry code: (i) $-x,-y+1,-z+1$.

Hydrogen-bond geometry $\left(A,{ }^{\circ}\right)$

\begin{tabular}{lllll}
\hline$D-\mathrm{H} \cdots A$ & $D-\mathrm{H}$ & $\mathrm{H} \cdots A$ & $D \cdots A$ & $D-\mathrm{H} \cdots A$ \\
\hline $\mathrm{O} 6-\mathrm{H} 6 \cdots \mathrm{O} 4^{\mathrm{i}}$ & $0.73(5)$ & $1.91(5)$ & $2.625(3)$ & $167(5)$ \\
$\mathrm{C} 5-\mathrm{H} 5 B \cdots \mathrm{I} 2$ & 0.98 & 3.01 & $3.987(3)$ & 172 \\
$\mathrm{C} 6-\mathrm{H} 6 B \cdots \mathrm{O} 5^{\mathrm{ii}}$ & 0.98 & 2.21 & $3.190(4)$ & 174 \\
$\mathrm{C} 12-\mathrm{H} 12 A \cdots \mathrm{O} 1$ & 0.98 & 2.59 & $3.561(4)$ & 173 \\
$\mathrm{C} 12-\mathrm{H} 12 B \cdots \mathrm{I} 1^{\mathrm{iii}}$ & 0.98 & 3.09 & $4.051(3)$ & 167 \\
$\mathrm{C} 14-\mathrm{H} 14 A \cdots \mathrm{I} 2^{\text {iv }}$ & 0.99 & 3.15 & $4.070(4)$ & 156 \\
$\mathrm{C} 17-\mathrm{H} 17 B \cdots \mathrm{I} 1^{\text {iv }}$ & 0.98 & 3.05 & $4.015(4)$ & 169 \\
$\mathrm{C} 16-\mathrm{H} 16 A \cdots \mathrm{I} 1^{\text {iii }}$ & 0.99 & 3.11 & $3.932(4)$ & 141 \\
$\mathrm{C} 24-\mathrm{H} 24 A \cdots \mathrm{O} 3^{\mathrm{i}}$ & 0.98 & 2.57 & $3.482(5)$ & 154 \\
$\mathrm{C} 28-\mathrm{H} 28 B \cdots 2^{\mathrm{v}}$ & 0.99 & 3.09 & $3.981(4)$ & 150 \\
$\mathrm{C} 30-\mathrm{H} 30 A \cdots \mathrm{O} 5^{\mathrm{vi}}$ & 0.98 & 2.57 & $3.404(5)$ & 143 \\
& & & &
\end{tabular}

Symmetry codes: (i) $-x,-y+1,-z+1$; (ii) $-x+1,-y+1,-z+2$; (iii) $x-1 / 2,-y+3 / 2, z+1 / 2$; (iv) $x-1, y, z$; (v) $x+1 / 2,-y+3 / 2, z+1 / 2$; (vi) $-x+2,-y+1,-z+2$. 